\title{
ZWISCHENSTAATLICHKEIT UND HERRSCHERIDEAL IN MILITÄRISCH-POLITISCHEN SACHBÜCHERN. EIN KOMParatistischer Versuch Über SŪN ZǏ, Kauțilya, Aineias Taktikos und Machiavelli
}

David Engels

Instytut Zachodni, Poznań

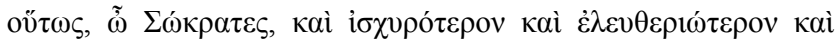

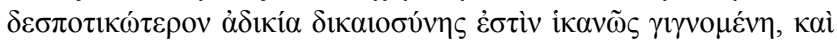

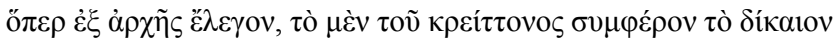

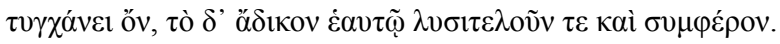

Plat., Pol. 344c-d

\begin{abstract}
All too often, the modern political sciences attribute the development of a purely pragmatic, non-idealistic state theory to Machiavelli, perhaps with some fleeting allusions to the importance of Plato, Aristotle or Polybius. However, a closer examination of our sources shows that many other civilisations have developed similar approaches, generally in periods experiencing political and social transformations not dissimilar to the complex evolutions of the European 16th century. In this paper, we will try to critically compare a series of authors in order to demonstrate the interest of a cross-cultural approach to the subject of state-theory, namely Sūn Ž̀, Kauṭilya, Aineias Taktikos and Machiavelli.
\end{abstract}

Key words: Sūn Ž̃, Kauṭilya, Aeneas Tacticus, Machiavelli.

\section{Einleitung}

Allzuoft stößt man in der modernen politologischen Literatur auf die Aussage, Machiavelli sei der weltweit erste, rein pragmatische Vertreter einer ausschließlich auf Effizienz und Rationalität ausgerichteten Staatstheorie, so daß die Diskussion über die Geschichte der diesbezüglichen Theoriebildung sich meist auf das Studium der letzten fünf Jahr- 
hunderte abendländischer Geistesgeschichte beschränkt. ${ }^{1}$ Nur gelegentlich wird dieser Ansatz, vor allem natürlich im Bereich der Altertumswissenschaft, durch einen kurzen Verweis auf die besondere Bedeutung der Antike korrigiert, doch selbst deren Denken wird meist ganz auf das idealistische bzw. enzyklopädische Staatsdenken eines Platon, Aristoteles oder Cicero verengt.

Das hierdurch indirekt postulierte Entwicklungsschema, welches, vereinfachend gesagt, von einer Genese rationeller, wenn auch angeblich naiv-idealistischer Staatlichkeit in der klassischen Antike ausgeht, sodann einen „Rückfall“ in wesentlich geistlich dominierte Formen gesellschaftspolitischen Denkens in Spätantike und Mittelalter annimmt und schließlich eine Wieder- oder sogar Neuentdeckung reiner, vorurteilsloser Staatlichkeit in der frühen Neuzeit postuliert, welche dann in den Rechtstaat der Moderne als Krönung der Schöpfung münde, ist weit verbreitet, zeichnet sich aber durch eine Linearität des Denkens und eine eurozentrische Teleologie aus, welche überaus problematisch sind; nicht nur, weil sie unwissentlich alte Vorurteile und Einseitigkeiten transportieren, sondern auch, weil sie in keiner Weise mehr mit dem heute erreichten, leider nur selten in seiner ganzen Fülle ausgewerteten universalhistorischen Forschungsstand übereinstimmen. Denn auch in China, Indien und vielen anderen vergangenen Hochkulturen haben sich zu verschiedensten Zeiten staatstheoretische Ansätze und Modelle entwickelt, welche es mit den oben erwähnten in jeder Beziehung aufnehmen können, und zwar nicht nur, was den eigentlichen Geist der Sache, sondern auch, was die Komplexität der Argumentation betrifft, ${ }^{2}$ wie wir im Folgenden kurz anreißen wollen.

Eine umfangreiche Analyse des diesbezüglichen Schrifttums selbst nur unter den wichtigsten staatstheoretischen Aspekten würde den gesetzten Rahmen natürlich bei weitem überschreiten; ${ }^{3}$ wir müssen uns daher im folgenden sowohl, was unsere Fragestellung, als auch, was unsere Materialbasis betrifft, stark begrenzen. Was die Fragestellung betrifft, so wollen wir uns hier auf einen bislang im interkulturellen Vergleich nur selten betrachteten Aspekt konzentrieren, nämlich den Stellenwert zwischenstaatlicher Beziehungen in ihrem expliziten oder impliziten inneren Bezug zum jeweiligen Herrscherbild; ein Punkt, der zwar in den behandelten Schriften meist eher sekundär diskutiert wird, in vielerlei Hinsicht aber von fundamentaler Bedeutung ist für die neue Definition von Grundlagen und Ziel der in den jeweiligen Untersuchungen aufgestellten „rationalen“ Staatstheorie. Was nun unsere textliche Ausgangsbasis angeht, so wurde entschieden, aus der breiten Masse des verfügbaren Materials jeweils die frühesten erhaltenen, für unser Thema relevanten Schriften zu berücksichtigen, nämlich (in ungefährer chronologischer Reihenfolge) das mit dem Namen des Sūn Zǐ verbundene bīng

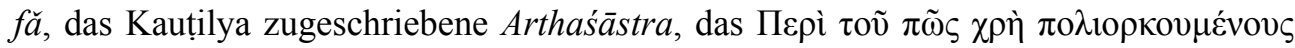

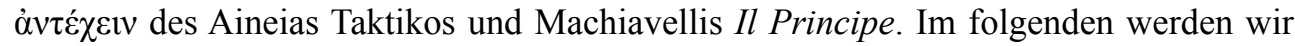

1 Vgl. z.B. die (ansonsten durchaus nützlichen) Darstellungen bei Vorländer 1926; Schrenck-Notzing 1978; Paret - Craig - Gilbert 1986; Paret 1986; Viroli 1999; Berridge - Keens-Soper - Otte 2001.

${ }^{2}$ Man darf hier nicht den Fehler begehen, die Geschichte der Staatstheorie gewissermaßen vom (scheinbaren) „Ende“ westlicher Rechtstaatlichkeit und verwaltungstechnischer Modernität zu denken: Es ist weder der Umfang eines Gesetzestextes, noch die technischen Mittel seiner Durchsetzung, welche bei einer komparatistischen Betrachtung, die sich über bloße Akzidentien erheben will, in Betracht kommen, sondern allein der Geist, der ihn bewegt.

${ }^{3} \mathrm{Zu}$ einer generellen Verortung der Wechselbeziehung zwischen Idealvorstellung und Wirklichkeit in der Ausübung von Macht vgl. die Einleitung zu Engels - Geis - Kleu 2010. 
in einem ersten Schritt versuchen, jeden einzelnen dieser Texte zunächst separat vorzustellen und auf die gewählte Thematik hin zu prüfen; in einem zweiten, abschließenden Schritt wollen wir dann eine allgemeine Vergleichung versuchen. ${ }^{4}$

\section{Zwischenstaatliche Beziehungen bei Sūn Ž̌, Kauṭilya, Aineias Taktikos und Machiavelli}

\subsection{Sūn Ž̃}

Das unter dem Titel „Kriegskunst“ (兵法, bīng fă) erhaltene Traktat, das traditionell meist Sūn Wǔ, besser bekannt als Sūn Ž̌ (孫子: „Meister Sūn“, ca. 544-496), ${ }^{5}$ zugeschrieben wird, ist nicht nur die erste vollständig erhaltene ${ }^{6}$ kriegswissenschaftliche Abhandlung der chinesischen Hochkultur, sie ist auch eine der ersten theoretischen Auseinandersetzungen Chinas mit grundlegenden Konzepten rationeller Staatlichkeit überhaupt, jedenfalls soweit man dies aus den Trümmern der klassischen chinesischen Literatur erschließen kann. ${ }^{7}$ Zwar befaßten sich auch viele Texte der vorangehenden Zeit mit dem Staat und seiner optimalen inneren wie äußeren Führung; ja, es ließe sich sogar formulieren, daß die gesamte chinesische Literatur, vielleicht von der Lyrik und der Fachliteratur abgesehen, nichts anderes sei als eine einzige, gewaltige Beschäftigung mit Fragen der bestmöglichen gesellschaftlichen Ordnung. Doch standen die diesbezüglichen Texte der vorherigen Zeit meist entweder unter dem Vorzeichen der Ritualistik, wie etwa die berühmten, trotz vielfältiger Redaktionsstufen wohl zumindest in Teilen bis in die westliche Zhōu-Zeit zurückreichenden „drei Ritualbücher““-das 周禮 $(z h o \bar{u}$ lì), das 儀禮 (yi lǐ) und das 禮記 ( $l \grave{l} j i)$ - oder aber unter dem Vorzeichen restaurativer, ideal-

${ }^{4}$ Ein solcher Vergleich ist bislang vor allem auf Basis des Arthaśāstra gemacht worden, dessen Einordnung als ,indischer Machiavelli“ gewissermaßen sprichwörtlich geworden ist (s. etwa pars pro toto Dikshitar 1927; Sil 1985a, 64-108; 1985b; Boesche 2002b). Über einige Oberflächlichkeiten wird dabei leider meist nicht wesentlich hinausgegangen, zumal der Vergleich meist rein bilateral bleibt und nur selten weitere Autoren anderer Kulturen heranzieht, und wenn, dann meistens solche, die sich eher durch ihre Klassizität oder ihren Bekanntheitsgrad auszeichnen als durch eine tatsächliche formale wie inhaltliche Analogie. Vgl. etwa Spengler 1969, der das Arthaśāstra mit Platons Staatsschriften und mit dem商君, dem書Shāng jūn shū vergleicht, Sil 2017, der die Analekten des Konfuzius diskutiert, oder Sil 1985a, das Arthaśāstra auch mit Platon (26-44) und sogar mit Aristoteles (45-63) zusammenbringt. Ähnliches ließe sich über die zeitgenössischen Versuche sagen, Sūn Ž̌ und das ihm zugeschriebene bīng fă zur Basis verschiedenster Management-Theorien zu machen und mehr oder weniger anachronistisch mit verschiedenen anderen Texten der Weltliteratur in Verbindung zu bringen.

5 Allg. zu Sūn Zĩ (bzw. dem ihm zugeschriebenen bīng fă) vgl. Lau 1965; Lau - Ching 1992; Mair 2007.

6 Allerdings ist hier zu erwähnen, daß das sich durchaus auf Vorgängerwerke beruft, deren Datierung, Umfang, Thematik, Autorschaft und vielleicht sogar bloße Existenz in vollständige Schatten gehüllt sind; s. z.B. das in Sūn Ž̌, bīng fă 7,23 belegte Buch „Heer und Angriff“ (軍政).

${ }^{7}$ Die grundsätzliche Problematik der Zerstörung vieler klassischer Schriften durch den ersten Kaiser ist bekannt; allerdings wird zum einen das Ausmaß dieser Vernichtung oft überschätzt, war es doch offensichtlich möglich, viele Schriften nach dem Zusammenbruch der kurzlebigen Qín-Dynastie wieder zu rekonstituieren (freilich in vielen Fällen mit signifikanten Abänderungen), während zum anderen neue Ausgrabungen zahlreiche klassische Schriften zutage gefördert und unser Quellenmaterial erheblich erweitert haben. 
staatlicher Gesellschaftsphilosophie, wie sie uns etwa in den vielfältigen Überlegungen des Kŏng Ž̃ (孔子, ca. 551-479) entgegentreten.

Der Verfasser des dem berühmten General Sūn Ž̌ zugeschriebenen, wohl im frühen 5. Jh. v.Chr. entstandenen bīng fă, scheint einer der ersten gewesen zu sein, ${ }^{8}$ der sich innerhalb des reichen chinesischen Schrifttums bemühte, sich abseits von traditionalistischen oder moralistischen Erwägungen ganz auf die realen Gegebenheiten der zeitgenössischen chinesischen Staatswelt zu konzentrieren und, hiervon ausgehend, ein rein auf Effizienz und Erfolg orientiertes Handbuch zu verfassen, was sich auch an der Einbeziehung historischer exempla in seine Ratschläge zeigt. ${ }^{9}$ Die politischen Gegebenheiten hierfür waren denkbar günstig: Nach dem endgültigen Zusammenbruch der Reichseinheit der Östlichen Zhōu, deren ehemaligen Lehnsmänner sich in der „Frühlings- und Herbstzeit“" (so genannt nach den gleichnamigen Annalen 春秋時代, chūnqiū shídài) zu unabhängigen Fürsten aufgeschwungen hatten, kam es ab dem frühen 5. Jh. in der Epoche der „Kämpfenden Staaten“ (ebenfalls so genannt auf Grundlage des gleichnamigen Geschichtswerks 戰國時代, zhànguó shídài) zu einer immer stärkeren Konzentration der Macht auf etwa sieben unabhängige Staaten, welche einander in wechselnden Bündnissen nahezu pausenlos befehdeten, bis es schließlich dem Staat Qín (秦) gelang, die Reichseinheit herzustellen. ${ }^{10}$ Auch intellektuell waren das 5. und 4. Jh. eine Umbruchszeit, welche die Zersplitterung der altererbten kultischen und moralischen Vorstellungen in die berühmten ,100 Schulen“ sahen, ${ }^{11}$ zu denen eben auch die ,Militaristen“" zählten, ${ }^{12}$ zu denen Sūn Ž̃ gehörte, bevor es dann mit der Reichseinigung und der Festigung der Hàn-Dynastie zur intellektuellen Hegemonie des Konfuzianismus kam.

${ }^{8}$ Die Datierung des Werkes ist, ebenso wie seine Zuschreibung zu Sūn Zĩ, bis heute umstritten. Sīmă Qiān berichtet im shĭjì (65.2161-2162, ed. Nienhauser) von einem militärwissenschaftlichen Traktat des Sūn Wǔ in 13 Kapiteln, das von Ho Lu, König von Wú (514-496), gelesen worden war: 孙子武者，齐人也。 以兵法见于吴王阖庐。阖庐曰：“子之十三篇，吾尽观之矣，可以小试勒兵乎? ”对曰：“可。”(Sūn Ž̌ Wǔ war aus Qí gebürtig. Er wurde zu einer Audienz bei Ho Lu, König von Wú, geladen wegen seiner Kriegskunst. Ho Lu sagte: 'Ich habe alle Eure dreizehn Kapitel gelesen. Könnt Ihr mir ein kleines Beispiel Eurer Kunst geben, Truppen zu drillen?' 'Ich kann', antwortete er.) Hierauf forderte der König, Sūn Zǐ solle seine Hofdamen drillen, was dieser, nachdem er bei der ersten Zuwiderhandlung die beiden Offizierinnen gegen den Widerspruch des Königs enthauptet lassen hatte, glänzend vollbrachte.

Der Text, nachweislich im 2.-3. Jh. n.Chr. ediert und bearbeitet wurde seit dem 13. Jh. eher der Zeit der reifen „Kämpfenden Staaten“ zugeordnet und sogar Sūn Wǔs Nachfahren Sūn Bìn (孫臏) zugeschrieben, der im 4. Jh. v.Chr. wirkte. Ein Manuskriptfund des Jahres 1972 hat allerdings in einem Grab der Zeit zwischen 134 und 118 v.Chr. nicht nur Fragmente des bīng fă des Sūn Zǐ zutage gebracht, welche eine Korrektur der bislang erhaltenen Version erlaubte und Licht auf die komplexe Textgeschichte geworfen hat, sondern auch des gleichnamigen bīng fă des Sūn Bìn, das sich in vielem auf die erste Schrift beruft (vgl. Lau - Ames 2003). Dies hat dazu geführt, daß der Text des Sūn Zǐ heute meist in die erste Hälfte des 5. Jh. datiert wird. Inwieweit er zumindest im Kern tatsächlich auf den General des späten 6. Jh. zurückgeführt werden kann, muß unsicher bleiben. Vgl. allg. Ames 1993; zum Kontext des Manuskriptfundes auch Loewe 1977.

${ }^{9}$ Freilich gilt es hier zu bedenken, daß die chinesische Literatur seit jeher stark von der Spannung zwischen allgemeinen theoretischen Überlegungen und ihrer Verdeutlichung am konkreten Fallbeispiel lebte.

${ }^{10}$ Zum historischen und ideologischen Kontext s. Hsu 1965; Li 1985; Loewe - Shaughnessy 1999; McNeal 2000.

${ }^{11}$ Allg. einführend zu den 100 Schulen vgl. Graham 1993; Bauer 2001; Cheng 2002; Denecke 2011.

${ }^{12}$ Allg. zur „Militärschule“ vgl. die Textausgabe der berühmten „Sieben Klassiker“ bei Sawyer 2007. 
Zwar handelt der Großteil des mit dem Namen Sūn Zì verbundenen bīng fă von militärischen und strategischen Fragen und scheint auf den ersten Blick für unsere eigene Fragestellung nur von bedingtem Interesse zu sein. Tatsächlich aber gründet die gesamte Anlage des Werkes auf einer Reihe staatstheoretischer und zwischenstaatlicher Grundlagen, die es für uns auch abseits seines Ranges als frühestem militär- und staatstheoretischen Schriftstück Chinas von höchstem Wert scheinen lassen. ${ }^{13}$ Schon die Struktur in 13 Kapiteln zeigt die thematisch überaus breite Anlage der Abhandlung, geht es doch in Kapitel 1 um die optimale Vorbereitung, in Kapitel 2 um die Analyse der Situation, in Kapitel 3 um die Vermeidung des Kriegs durch Diplomatie, in Kapitel 4-8 um den richtigen Einsatz psychologischer und logistischer Machtmittel, und in Kapitel 9-13 um Einzelfragen zur Einschätzung von Terrain, Situation und feindlicher Absicht.

In unserem Sinne interessant ist zunächst die argumentative Ausgangsbasis des Werkes. Die Existenz zahlreicher, einander befehdender Staaten, welche in Kämpfe verstrickt sind, welche vom nackten Überlegen bis zur Wiederherstellung einer gesamtchinesischen Einheit reichen, wird als unwiderruflich gegeben hingenommen, ebenso wie die Natur des Menschen als eines unbeständigen, vor allem von seinen Trieben kontrollierten Wesens:

Sūn Zĩ sprach: Der Krieg ist für einen Staat eine bedeutende Angelegenheit, denn er entscheidet über Leben und Tod und kann zum Untergang oder Weiterbestehen eines Staates führen. Auf jeden Fall ist Krieg eine Angelegenheit, die einer genauen Untersuchung unterzogen werden muß. ${ }^{14}$

Ziel des Werks ist daher weder eine ideale Friedensordnung, wie sie noch Kŏng Zì vorschwebte, noch eine Verrechtlichung oder gar Moralisierung des Krieges, wie seine Nachfolger sie betreiben sollten: Im Mittelpunkt stehen bei Sūn Zǐ ausschließlich Überlegungen, mit welchen Mitteln ein optimal geführter Krieg zum Sieg und somit zur Ausdehnung der Macht des Siegers führen kann. Bezeichnenderweise wird „Krieg“ hier daher keineswegs nur in einem rein technischen Sinne begriffen, sondern vielmehr als Oberbegriff für eine jegliche, gegen den Feind gerichtete strategische Maßnahme:

Hundert Schlachten zu schlagen und hundert Siege zu erringen, ist nicht ein Zeichen von Perfektion. Wer den Feind ohne Schlacht besiegt, versteht sich wirklich auf die Kriegführung. Die beste Angriffsstrategie ist, die Allianzen zu zerschlagen, dann die Armee und zuletzt die Städte. ${ }^{15}$

Ziel dabei ist zuerst Selbsterhaltung, dann Ausdehnung und schließlich, wie angesichts der breiten Präsenz dieses Topos in der zeitgenössischen Literatur wohl anzunehmend ist, die Rekonstitution der Einheit Chinas. ${ }^{16}$

Wie auch immer geartete moralische, rechtliche oder religiöse Vorstellungen gelten somit als reines Hindernis auf der Suche nach dem Sieg: Im Krieg ist alles erlaubt, das dem Sieg dient, selbst mögliche Warnungen der Götter müssen in den Wind geschlagen

${ }_{13}$ Allg. zum staatstheoretischen Kontext jener Zeit Vandermeersch 1965; Li 1977; Ames 1994; Pines 2002; Pines 2009b.

${ }^{14}$ Sūn Zĩ, bing fă 1,1-2: 孫子曰：兵者, 國之大事, 死生之地, 存亡之道, 不可不察也. Sūn Zǐ soll im folgenden nach der Übersetzung von Eisenhofer (2014) zitiert werden.

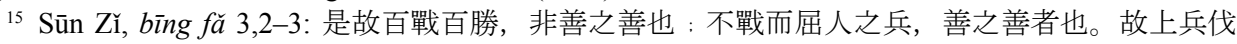
謀其次伐交，其次伐兵，其下攻城。攻城之法為不得已。

${ }^{16}$ Zur Idee der „Einheit“ Chinas vgl. Pines 2009a. 
werden, wenn sie die Truppenmoral schwächen, und individualistischere Formen von Religion daher streng kontrolliert werden: ${ }^{17}$

Verbiete deinen Männern, das Orakel zu befragen, zerstreue die Zweifel, und sie werden dir bis in den Tod folgen. ${ }^{18}$

So lesen wir denn auch bei Sūn Ž̃: „Krieg ist Täuschung”19, was durch eine Fülle von Kriegslisten untermauert wird. Das bing fă richtet sich dabei interessanterweise nicht an einen bestimmten Staat (und wurde ja auch, sollte es tatsächlich dem historischen Sūn Ž̃ zuzuschreiben sein, offensichtlich in einer Situation verfaßt, als dieser als politisch unabhängiger Gelehrter und Fachmann, als shi, 士, den Herrschern seiner Zeit seine Dienste anbot) ${ }^{20}$ sondern scheint als allgemeines, von jeglicher persönlichen und politischen Loyalität losgelöstes Fachbuch konzipiert worden zu sein. Dies impliziert, daß die pragmatische und amoralische, rein erfolgsorientierte Vorgehensweise keineswegs als Besonderheit des potentiellen Adressaten des Werks scheint, sondern vielmehr als allgemeiner Charakterzug eines jeden auf Überleben und Ausdehnung orientierten Staates. Hieraus erklärt sich, daß die zahlreichen Spionage- und Sabotagetechniken natürlich auch dem jeweiligen Gegner unterstellt werden, dessen potentielle Spione es zu Beginn eines Krieges auszuschließen gilt:

An dem Tag, an dem du angreifen wirst, sperre alle Grenzpässe, zerbrich alle Kennzeichen und laß keine Gesandten des Feindes mehr passieren. ${ }^{21}$

In diesem Sinne wird auch eindringlich vor möglichen Friedensangeboten des Gegners als einer reinen Kriegslist gewarnt, was im Umkehrschluß natürlich die Frage aufwirft, inwieweit unter diesen Bedingungen überhaupt noch ein tatsächlicher Friedensvertrag zustande kommen kann, der nicht ganz auf Unterwerfung und Vernichtung beruht:

Sanfte Worte und erhöhte Vorbereitungen deuten auf ein Vorrücken hin. Gewaltsame Worte und vorpreschende Reiter zeigen seinen Rückzug an. [.....] Die Bitte um Frieden ohne Vertrag ist ein Zeichen von List. [...] Kommen Gesandte mit unterwürfigen Worten, wünscht der Feind eine Ruhepause. ${ }^{22}$

Gleichzeitig folgt aus diesem Ansatz auch ein tiefes Mißtrauen selbst gegenüber einem einmal erreichten Friedenszustand, da dieser immer nur als vorübergehende Unterbrechung des natürlichen, also kriegerischen Zustands betrachtet werden kann. Die reine Zweckorientierung des Werks führt Sūn Ž̌ logischerweise auch zu einem tiefen Mißtrauen vor jeder Form von Bündnis mit einem fremden Staat, in dem er naturgemäß nur den nächsten Feind zu erblicken vermag. Zwar ist sich der Autor der Notwendigkeiten von Bündnissen durchaus bewußt, sobald das Risiko überschaubar ist (,Deshalb sollst du keine Allianz eingehen, wenn du die Pläne und Absichten der anderen Fürsten nicht

${ }_{17}$ Zu den religiösen Vorstellungen dieser Zeit vgl. Ching 1993; Lagerwey 2004; Lagerwey - Kalinowski 2008; Nadeau 2012; Pankenier 2013; Pregadio 2013.

${ }^{18}$ Sūn Zĩ , bing fă 11,26: 禁祥去 疑, 至死無所之。

19 Sūn Zì, bing $f a ̆$ 1, 18: 兵者, 詭道也。

${ }^{20}$ Zur gesellschaftlichen Gliederung dieser Zeit s. von Falkenhausen 2006.

${ }^{21}$ Sūn Ž̌ , bing fă 11,63: 是故政舉之日，夷關折符，無通其使 :

${ }^{22}$ Sūn Ž̌ , bing $f a ̆ ~ 9,24 ; 26 ; 38:(24)$ 辭卑而備者, 進也：辭強而進驅者, 退也： (26) 無約而請和 者, 謀也：(38) 來委謝者，欲休息也。 
kennst. ${ }^{\text {(“23) }}$, aber trotzdem sind sie als Mittel der Friedenswahrung bzw. Kriegsleistung geringer einzuschätzen als unabhängiges, rasches und energisches Handeln:

Deshalb kämpft er weder um Verbündete für sein Reich, noch stärkt er die Macht anderer Reiche. Indem er den Feind überwältigt, kann er dessen umwallte Städte einnehmen und das Land unterwerfen. ${ }^{24}$

Diesem desillusionierten Politikbild des Autors entspricht ein analoges Bild vom Menschen und vor allem vom Herrscher bzw. Feldherr. Dieser muß sich möglichst von allen schwächenden menschlichen Regungen freizuhalten wissen, zu denen nicht nur Moralität oder zu ausgeprägtes Rechtsgefühl zählen, sondern auch übertriebene Sorge um das eigene Volk, welche alle dem effizienten Einsatz der jeweiligen Machtmittel nur im Wege stehen könnten:

Für einen General gibt es fünf Gefahren. Ist er todesmutig, kann er leicht getötet werden. Hängt er zu sehr am Leben, ist er leicht gefangen zu nehmen. Ist er von aufbrausendem Temperament, kann er schnell beleidigt werden. Ist er zu ordentlich und zu aufrichtig, fühlt er sich zu schnell entehrt, und ist er zu besorgt um seine Männer, ist er leicht in Schwierigkeiten zu bringen. ${ }^{25}$

Das dieser Sichtweise zugrundeliegende Mißtrauen erklärt zweifellos auch, wieso Sūn Ž̀, bezeichnenderweise ganz im Gegensatz zu Clausewitz, den idealen chinesischen Feldherrn geradezu dazu auffordert, die sich ihm gegebenen Vorteile um jeden Preis auszunutzen, selbst wenn er von seinem jeweiligen Herrscher andere Instruktionen erhalten hat; ein Primat der Politik vor dem Krieg wird hier also bewußt abgelehnt:

Wenn der oberste Grundsatz beim Krieg der Sieg ist, kämpfe, selbst wenn der Herrscher befiehlt, nicht zu kämpfen. Ist das Ziel nicht der Sieg, und der Herrscher befiehlt zu kämpfen, dann greife nicht an. Ein General, der nicht um des Ruhmes willen vorrückt und sich zurückzieht, ohne Schande zu fürchten, der nur daran denkt, das Volk zu schützen und Vorteil für den Herrscher zu suchen, ist ein Juwel für den Staat. ${ }^{26}$

Es bedarf daher nur wenig Vorstellungskraft, sich das Auskommen der sich aus solchen inneren Konflikten ergebenden Dispute zwischen dem König und seinem Feldherrn auszumalen, und es ist wohl keine Überraschung, daß gerade in jener Zeit der chinesischen Geschichte mit ihrer gewaltigen sozialen Mobilität ${ }^{27}$ nicht nur zahlreiche Usurpationen auf der Tagesordnung standen, sondern auch die zeitgenössischen Staatstheoretiker das Modell der freiwilligen Abdankung des Herrschers zugunsten des am besten geeigneten Staatsdieners propagierten... ${ }^{28}$

${ }^{23}$ Sūn Ž̃, bing fă 7,12: 故不知諸侯之謀者, 不能豫交:

${ }^{24}$ Sūn Zĭ, bīng fă 11,55: 是故不爭天下之交, 不養天下之權, 信己之私, 威加于敵, 則其城可 拢, 其國可雄

${ }^{25} \operatorname{Sūn} Z \mathrm{Z}, \operatorname{bin} g f a ̆$ 8,12: 故將有五危：必死，可殺也；必生，可虜也：忿速，可侮也：廉潔，可辱 也：愛民，可煩也。

${ }^{26}$ Sūn Zǐ, bīng fă 10,23-24: 故戰道必勝, 主曰無戰, 必戰可也：戰道不勝, 主曰必戰, 無戰可也 故進不求名，退不避罪，惟人是保，而利合于主，國之寶也。

${ }^{27} \mathrm{Vgl}$. allg. Hsu 1965.

${ }^{28} \mathrm{Zu}$ diesen neuen Konzepten vgl. etwa das róng chéng shi (容成氏); hierzu Pines 2010. 


\subsection{Kauṭilya}

Das Arthaśāstra (अर्थशास्त्र; grob übersetzt die „Wissenschaft von der Politik“") ${ }^{29}$ ist die erste (erhaltene) staats- und militärpolitische Fachschrift der indischen Hochkultur und bis heute ein beredtes Zeugnis für die Komplexität und Rationalität des klassischen indischen Denkens. Freilich finden sich auch in den zeitlich aller Wahrscheinlichkeit nach früher zu datierenden Texten zahlreiche Überlegungen zu gesellschaftlichen und politischen Fragen, so etwa in der Bhagavad-gītā (भगवद्गीता), die in das 7. Buch der Mahābhārata (महाभारतम्) Eingang fand. Doch handelte es sich hierbei trotz aller Rationalität eher um stark philosophisch und idealstaatlich geprägte Überlegungen, deren Ziel es nicht war, größtmögliche machtpolitische Effizienz zu erzielen, sondern den Einzelmenschen mit dem Ganzen in Einklang zu bringen, und die dementsprechend auch das Scheitern an der Umwelt als vorteilhafter als den Verrat am eigenen Heil betrachten.

Die Entstehungszeit des Arthaśāstra ist bis heute hoch umstritten. Zwar gibt der Text selber vor, ${ }^{30}$ nur die Ansichten des Kauțilya (कौटलिय; ca. 350-283) wiederzugeben, ${ }^{31}$ doch ist die Situation erheblich komplizierter. Kautilya, auch unter den Namen Viṣnugupta und Cāṇakya bekannt, war Berater und Minister des bei den Griechen als Sandrakottos bekannten Maurya-Herrschers Candragupta (चन्द्रगुप्त, regierte ca. 321298), dem nach der Absetzung der Nanda-Könige mit ihrer komplizierten Kollektivherrschaft auch faktisch die weitgehende politische Einigung der Ganges- und Indusebene gelang und somit die Errichtung eines den Großteil des hinduisierten Gebietes umfassenden Reichs. Kauṭilya soll auch noch dessen Sohn Binḍusāra (298-272; in den griechischen Quellen Allitrochades bzw. Amitrochates nach seinem indischen Beinamen अमत्रिघात, Amitraghāta, der „Feindestöter“) gedient haben und somit gewissermaßen die graue Eminenz hinter der ersten indischen Reichseinigung seit mythologischer Zeit gewesen sein. Tatsächlich allerdings ist das unter dem Namen Kauțilyas überlieferte Arthaśāstra zumindest in der heute vorliegenden Form ${ }^{32}$ wohl erst nach der Maurya-Zeit entstanden und zwischen dem 2. Jh. v.Chr. und dem 3. Jh. mehrfachen Überarbeitungen

${ }^{29}$ Allgemein zum Arthaśāstra vgl. Meyer 1926; Konow 1945; Schlingloff 1967; Scharfe 1968; Trautmann 1971; Choudhary 1971; Ritschl - Schetlich 1973; Parmar 1987; Kühnhardt 1988; Boesche 2002a; 2002b; Zaman 2006; McClish 2009; Trautmann 2012; Olivelle 2013; Gautam 2013. Einen kompletten Überblick über die frühere Forschung liefert Sternbach 1973.

${ }^{30}$ Kautilya, Arthaśāstra 15,1,37-38: येन शास्त्रं च शस्त्रं च नन्दराजगता च भूः । अमर्षेण उद्धृतान्याशु तेन शास्त्रं इदं कृतम् (Von dem Manne, der die Staatskunde und die Führung der Waffen und die Erde, als sie auf Nanda übergegangen war, in zorniger Ungeduld hurtig auf sichern und bequemen Boden heraushob, ist diese Staatskunde gemacht worden.) Die deutsche Übersetzung folgt der von Meyer 1926.

31 Zum historischen Kautilya und seinem Umfeld vgl. einführend Nilakanta Sastri 1967; Bhargava 1996; Boesche 2002a; 2002b. Sein Leben ist uns ausschließlich aus legendarischen Quellen bekannt, teils buddhistischen Ursprungs (das Mahavamsa), teils jainistischer (das Parishishtaparvan des Hemachandra); hierzu kommt dann noch ein Legendenkranz aus Kashmir (das Kathasaritsagara des Somadeva sowie das Brihat-Katha-Manjari des Ksemendra) sowie das Theaterstück Mudrārākṣasa des Viśākhadatta.

${ }^{32}$ Der Text ging im 12. Jh. verloren und wurde erst 1905 durch Rudrapatnam Shamasastry in einer auf Palmblätter geschriebenen Version wiederentdeckt und 1909 veröffentlicht, in der Folgezeit durch weitere Manuskriptfunde aber ergänzt und korrigiert. Die neueste Edition des Sanskrit-Textes ist Kangle 1969; die neueste Übersetzung Olivelle 2013. Für den Hausgebrauch weiterhin nützlich ist allerdings die zweisprachige Ausgabe von Narain 2005. 
und Ergänzungen unterzogen worden. Erst im Laufe dieser Zeit wurde der Text daher wohl auch mit dem Namen des halbmythisch gewordenen Kauțilya verbunden, ${ }^{33}$ der zu einer Chiffre für eine gesamtindische Einigung avanciert war, wie sie schließlich von den Gupta erreicht wurde, unter denen der Text auch zumindest formal seine endgültige Gestalt erhielt. Wenn wir daher auch nicht völlig ausschließen können, daß einige Elemente der Schrift tatsächlich auf Gedanken der prä-Maurya-Zeit zurückgehen, dürften viele doch eher die Realität der post-Maurya-Epoche der Śunga (शुडग्ग) wiederspiegeln, welche ebenfalls von einem breiten Spektrum verschiedenster, einander bekriegender Mächte geprägt war, die aber nun, anders als in der früheren Zeit, nicht nur beständig vom Wunsch beseelt waren, die verlorene Reichseinheit wiederherzustellen, sondern die auch durch den Handel mit dem Ptolemaier- und Seleukidenreich wie auch den Kontakt mit den zentralasiatischen Nomaden kulturell, wirtschaftlich und politisch breit an die auswärtige Welt angebunden waren ${ }^{34}$ so daß der Kampf um die Vorherrschaft eine völlig neue Dimension erhielt.

Das inhaltliche Spektrum des Arthaśāstra ist überaus vielfältig, und wenn es auch schwerpunktmäßig mit Fragen der Innenpolitik und der Kriegführung beschäftigt ist, beinhaltet es doch zahlreiche Ausführungen zu Fragen des Rechts, der Wirtschaft, der Diplomatie, der Philosophie sowie der Naturwissenschaften. Dies zeigt schon ein Blick auf die Struktur des Werkes, das in 15 Bücher (ungleichmäßig unterteilt in 150 Kapitel und 180 Themen) gegliedert ist, die sich mit so verschiedenen Fragen befassen wie der Erziehung des Fürsten (1-18), der Tätigkeit seiner Verwalter (19-56), dem Recht (57-75), dem „Ziehen der Dornen“ (76-88), dem Geheimnis (89-95), der Deutung auBenpolitischer Beziehungen in Form von konzentrischen Kreisen (96-97), der ,sechsteiligen" Strategie (98-126), den Gründen von Katastrophen (127-134), der Vorbereitung für den Krieg (135-146), dem Krieg selbst (147-159), den Beziehungen zu Föderalstaaten (160-161), den schwachen Herrschern (162-170), der Belagerung (171-176), magischen Praktiken (177-179) und schließlich einem allgemeinen Rückblick (180).

Uns soll es im Folgenden nun wesentlich, wenn auch nicht ausschließlich um die letzten Kapitel des Arthaśästra gehen, welche dem Krieg gewidmet sind und sich auch in ihrer Kürze und Dichte deutlich von den erheblich längeren ersten Kapiteln des Werks abheben, welche eher innenpolitischen und organisatorischen Fragen gewidmet sind.

Auch im Arthaśästra geht es, wie in Sūn Zǐs bing fă, um reine Optimierung und Effizienzsteigerung, freilich unter besonderer Berücksichtigung der Innenpolitik. Rechtliche oder gar moralische und religiöse Fragen spielen keinerlei Rolle, jedenfalls, insofern die souveränen Handlungen des Herrschers betroffen sind, der ausschließlich nach dem Gesichtspunkt seiner Machterhaltung zu agieren hat. Politik ist in diesem Sinne der institutionalisierte Sieg der Starken über die Schwachen; Recht ein Machtinstrument, kein

${ }^{33}$ Wenn auch letzte Sicherheit nicht zu erreichen ist, scheint der Text, dessen Grundlage wohl eine mündliche Lehre war, die auf die politischen Erfahrungen der post-Maurya-Zeit im 3. und 2. Jh. zurückging, zuerst zwischen dem 2. Jh. v.Chr. und dem 1. Jh. n.Chr. schriftlich niedergelegt, dann zwischen dem 1. und 2. Jh. v.Chr. ein erstes Mal wesentlich überarbeitet (wohl unter dem Titel Dandanīti) und schließlich zwischen dem späten 2. Jh. und $300 \mathrm{n}$.Chr. einer definitiven Redaktion unterzogen worden zu sein. Allg. hierzu Olivelle 2013, gestützt auf Trautmann 1971; McClish 2009 und Trautmann 2012.

${ }^{34}$ Zur Śunga-Zeit und zum allgemeinen historischen Kontext vgl. einführend Drekmeier 1962; Olivelle 2006; Singh 2015 und die klassische, wenn auch nunmehr veraltete Studie von Raychaudhuri 1927. Zur Stellung der im Arthaśāstra erwähnten Institutionen vgl. Sharma 1959 und Singh 2003. 
Regierungszweck; der Krieg nur die ultima ratio, wenn auch der Frieden nicht als Ruhezustand, sondern als eine Art „Krieg mit anderen Mitteln“ interpretiert wird, wie die Staffelung der ,sechs Strategien“ zeigt:

Es liegt hier doch ein sechsfaches Verfahren vor wegen der Verschiedenheit der Zustände. So Kauțilya. Dabei ist die Bindung durch Vertrag Friede, Zufügung von Schaden Krieg, ruhiges Zusehen Abwarten, Erstarkung und Erhebung Losmarschieren auf den Feind, sich einem anderen Hingeben Schutzsuchen. Die Hand auf beiden: Krieg und Frieden halten ist Doppelspiel. ${ }^{35}$

Ultimativer Zweck der Politik ist die Eroberung der „Welt“, womit im indischen Kontext wesentlich die politische Vereinigung der Ganges- und Indus-Ebene verstanden wird, denn wenn Indien auch, hierin der griechischen Welt nicht unähnlich, keine frühzeitliche politische Einigung gekannt hat, sollte doch der Gedanke einer inneren Zusammengehörigkeit der vedischen Völker seit jeher das Denken des Subkontinents bestimmen und nicht nur in der Bezeichnung Cakravārtin (चक्रवर्ता) eine feste Umschreibung erhalten, ${ }^{36}$ sondern auch zu vielfältigen Legendenbildungen reißen, unter denen v.a. die Figur des mythischen Vikramāditya (वक्रिमादत्यि) hervorragt. ${ }^{37}$

Und hat er die Erde erobert, dann nutznieße er sie gemäß den ihm zukommenden Rechten und Pflichten, indem er den verschiedenen Kasten und Lebensstufen ihre richtige Stelle anweist. ${ }^{38}$

Dieser Argumentationsansatz betrifft natürlich auch die zwischenstaatlichen Beziehungen, die rein auf Grundlage des Nützlichkeits- und Expansionsprinzip zu werten sind. Das Arthaśāstra ist dafür bekannt, als eine der ersten Schriften aus dem uralten Gedanken, daß ein Herrscher den Feind seines Feindes als seinen Freund zu betrachten hat, eine weitläufige Theorie konzentrisch angeordneter Bündnisstrukturen entwickelt zu haben. ${ }^{39}$ Doch selbst innerhalb der sich hieraus ergebenden Folgerungen ergibt das Traktat sich nie einem starren Schematismus, sondern lädt dazu ein, jede machtpolitische Situation nur auf Grund der ihr innewohnenden Verhältnisse zu betrachten. So heißt es denn auch:

Wird er schwächer als der Feind, dann schließe er einen Friedensvertrag. Erstarkt er und erhebt sich höher, dann erkläre er Krieg. Sieht er (wenn der Krieg erklärt ist): „Weder kann der Feind mich niederschlagen, noch ich ihn," dann warte er ab. Hat er das Übergewicht an den nötigen Dingen, dann rücke er gegen (den Feind) vor. Ist er geringer an Macht, so suche er eine Stütze. Kann das Werk nur mit Hilfe eines Genossen ausgeführt werden, dann mache er sich an das Doppelspiel. Dies ist die Begriffsbestimmung der sechs Verfahrensarten. ${ }^{40}$

${ }^{35}$ Kautilya, Arthaśāstra 7,1,5-6: षाड्गुण्यं एव एतद् अवस्थाभेदाद् इतक्कौटलियः । तत्र पणबन्धः सन्धई अपकारो वग्रिहः, उपेक्षणं आसनम्, अभ्युच्चयो यानम् परार्पणं संश्रयः , सन्धविग्रिह उपादानं द्वैधीभावः इतर्त षड्गुणाः ।

${ }^{36}$ Zum Konzept der „Weltherrschaft“ und der Unterschied zwischen Indern und „Barbaren“( s. Indra 1957.

${ }^{37}$ Zum Ideal der Weltherrschaft und des Vikramāditya im indischen Denken, das bis in die Gupta-Zeit lebendig sein sollte, vgl. Agrawal 1989, 174-176.

${ }^{38}$ Kauțilya, Arthaśästra 13,4,36: जत्विवा च पृथर्वीं वभिक्तवर्णाश्रमां स्वधर्मेण भुज्जीत

${ }_{39}$ Zur Bündnistheorie Kautilyas und den diplomatischen Gepflogenheiten seiner Zeit, vgl. Nag 1923; Mukherjee 1976; Roy 1981.

${ }^{40}$ Kautilya, Arthaśästra 7,1,6: परसमाद्द् हीयमानः सन्दधीत । अभ्युच्चीयमानो वगृह्णीयात् । न मां परो नाहं परं उपहन्तुं शक्तः इत्यासीत । गुणातशिययुक्तो यायात् । शक्तहिीनः संश्रयेत । सहायसाध्ये कार्ये द्वैधीभावं गच्छेत्। 


\section{Und:}

Mit einem Fürsten, der ihm gleich, und mit einem, der stärker ist, soll er einen Friedensvertrag schließen. Mit einem schwächeren soll er Krieg führen. Denn führt er Krieg mit einem stärkeren, dann geht er zur Schlacht wie zum Fußkampf mit einem Elephanten. Führt er Krieg mit einem gleichstarken, dann verursacht er Vernichtung auf beiden Seiten, wie wenn ein ungebranntes Gefäß mit einem ungebrannten zusammenschlägt. Einzig wer mit einem schwächeren Krieg führt, erlangt Erfolg, wie wenn ein Stein auf einen (irdenen) Krug trifft. ${ }^{41}$

Religiöse, moralische oder rechtliche Gesichtspunkte sucht man daher vergebens, und wenn das Arthaśāstra sich auch nirgendwo explizit gegen traditionelle Pfeiler der Gesellschaftsordnung wie die Kastenordnung ${ }^{42}$ mit ihren rechtlichen Konsequenzen oder der Ehrwürdigkeit diverser religiöser Würdenträger ausspricht und klar einen gesellschaftlichen Konservatismus bevorzugt, ${ }^{43}$ so entspringt dies nicht etwa der eigenen inneren Befürwortung dieser Institutionen, sondern vielmehr dem rein pragmatischen Ansatz des Werkes, welches eben nur auf Grundlage der jeweiligen Gegebenheiten operiert. So wird der Text nicht nur nicht müde, in hundertfacher Abwandlung den großen Nutzen von Spionen zu loben, welche unter anderem auch in der Verkleidung spiritueller Lehrer und Asketen operieren sollen, sondern befürwortet auch die Manipulation der Götterzeichen in kriegerischem Kontext, ${ }^{44}$ nicht unähnlich dem Ansatz bei Sūn Zĩ:

Ist die Schlachtaufstellung vollständig in Ordnung, dann soll er seinen Trupp von Wahrsagern usw. durch die Verkündigung, daß sowohl ein Allwissender (d.h. der König selbst) als auch die Macht der Götter (das Schicksal) mit ihnen sei, die eigenen Leute anfeuern und die feindliche Partei in Schrecken setzen. ${ }^{45}$

Wenn es im bìng fă hieß, „Krieg ist Täuschung”, ${ }^{46}$ gilt dies für das Arthaśāstra in fast schon monomanischem Ausmaß: Ein Großteil des Buches ist verschiedensten Formen von Spionage, Sabotage, Mord, Vergiftung, Betrug oder dem Anstiften zu diversen Zwistigkeiten gewidmet, die teils so abenteuerlich wirken, daß ihre tatsächliche Machbarkeit oft ebenso fragwürdig scheint wie die Wirksamkeit der verschiedenen, gegen Ende des Werkes aufgezählten Gifte und Tränke. Hierbei hat sich der Einsatz von Spionen nicht nur gegen den Feind, sondern auch das eigene Volk und selbst die eigene Verwaltung zu richten, wie das Arthaśāstra nicht müde wird zu betonen. Dementsprechend wird auch zu einem teils diplomatischen, teils rabiaten, immer aber skrupellosen Umgang mit möglichen inneren Feinden aufgerufen:

Ein Aufruhr der ihm Zunächststehenden ist schlimmer als ein Aufruhr der Außenstehenden. So ist schon vorher gesagt worden. Ein Aufruhr, der von irgendeinem dieser vier: Ratgeber, Haus-

${ }^{41}$ Kautilya, Arthaśästra 7,31: समज्यायोभ्यां सन्धीयेत, हीनेन वगृहणीयात् । वगृहीतो हज्जियायसा हस्तनि पादयुद्धं इवाभ्युपैती समेन चामं पात्रं आमेनाहतं इव उभयतः क्षयं करोती।

${ }^{42}$ Zur Situation der Kastenordnung in jener Zeit, als das Varṇa-System von der Jāti-Gliederung überlagert wurden, vgl. Dumont 1966; Gupta 1980 und Béteille 1996. Spezifisch zum Recht im Arthaśāstra s. Meyer 1927.

${ }^{43}$ Zur gesellschaftlichen Struktur der Zeit, in welcher die Grundlagen des Arthaśāstra geschaffen worden waren, vgl. Das 1994.

${ }^{44}$ Allg. hierzu Sharma 1954.

${ }^{45}$ Kauțilya, Arthaśāstra 10,3,15: पुनर्|आवर्तमानस्य नरिशस्य च जीवति । अधार्यो जायते वेगः तस्माद् भग्नं न पीडयेत् I S. ähnl. Kauțilya, Arthaśästra 10,3,18.

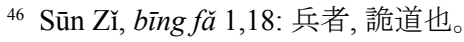


priester, Feldmarschall oder Kronprinz ausgeht, ist ein Aufruhr der Zunächststehenden. Den soll er durch Aufgeben seiner eigenen Fehler oder Verschuldungen oder nach Maßgabe der Macht und des Vergehens des anderen bewältigen. Dem Hauspriester gegenüber liegt, auch wenn er ein großes Verbrechen begangen hat, die Abhilfe (nur) in der Gefangensetzung oder in der Entfernung, gegenüber dem Kronprinz-Mitregenten in Gefangensetzung oder Tötung, falls ein mit Vorzügen begabter anderer Sohn da ist. ${ }^{47}$

Es ist somit nicht erstaunlich, daß auch die zwischenstaatlichen Beziehungen von keinem anderen Gesichtspunkt als dem des Mißtrauens bestimmt sind. Hilfstruppen sind daher, ebenso wie fremde Söldner, nach Möglichkeit zu vermeiden, weil ihnen naturgemäß schädliche Absichten unterstellt werden:

Und immer (die in der Aufzählung) vorhergehende Truppenart ist besser (als die ihr folgende), wo es gilt, sie für die Schlacht zu rüsten (sie in den Kampf zu schicken). Die angestammten Truppen sind besser als die Söldnertruppen, weil sie von des Herrschers Dasein das Dasein haben, beständig hergerichtet sind und ihm (immer treu) nachfolgen (was auch sein Schicksal sei). Weil man die Söldnertruppen immer unmittelbar zur Hand hat, sie rasch gefechtsbereit sind und sich dem Willen (des Königs und der Heeresleitung) gut fügen, so sind sie besser als die Verbandstruppen. Weil die Verbandstruppen vom eigenen Lande (des Fürsten) kommen, um ein und derselben Sache willen eingetreten sind, und Wettbewerb, Rachezorn, Glück und Gewinn, gemeinsam haben, so sind sie besser als die Freundestruppen. Weil die Freundestruppen im Hinblick auf Ort und Zeit nicht eingeschränkt sind (sondern zu allen Zeiten und allen Orten des Feldzugs mitkämpfen, während die vom Feinde kommenden Truppen z.B. gegen ihre Landsleute nicht in den Kampf gestellt werden dürfen), und weil sie um ein und derselben Sache willen herbeigekommen sind, so sind sie besser als die vom Feinde kommenden Truppen. Weil die vom Feinde kommenden Truppen von Ariern geleitet werden, so sind sie besser als die Waldstammtruppen. Diese beiden haben die Plünderungsbeute als Zweck im Auge. Gibt es nichts zu plündern oder kommt ein Mißstand (ein Unheil, eine Niederlage usw.), dann wird von beiden Schlangengefahr drohen (d.h. dann sind sie wie eine Schlange im Haus). ${ }^{48}$

Im selben Sinne gilt es vor allem, stärkere Staaten als Bündnispartner zu scheuen, um spätere Abhängigkeiten zu vermeiden; eine rein machtpolitische Strategie, welche etwa Fragen des Rechtes, der Verwandtschaft oder der Religion, welche ja durchaus zu ungleichen Bündnissen führen können, völlig ausklammert:

Er stelle sich unter den Schutz eines Fürsten, dessen Macht hervorragt über die Macht des (feindlichen) Nachbars. Ist keiner da, der stärker ist als dieser, dann begebe er sich unter seinen (des feindlichen Grenznachbarn) Schutz und bemühe sich dann, außer Sicht ihm durch irgendeines von den dreien: Schatz, Heer oder Land einen Dienst zu leisten. Denn ein großer Übelstand ist es für Könige mit einem, der an Stärke hervorragt, verbunden zu sein, außer wenn er selber von einem Feinde gepackt ist. ${ }^{49}$

${ }^{47}$ Sūn Ž̀, bìng fă 1,18: 兵者, 詭道也。

${ }^{48}$ Kautilya, Arthaśästra 9,3,5-6: मन्त्रपुरोहतिसेनापतयुिर्वाजानां अन्यतमकोपोऽभ्यन्तरकोपः तं आत्मद Kautilya, Arthaśāstra 9,2,12-17: र्वं पूर्वं च एषां श्रेयः सम्नाहयतुम् । तद्भावभावत्विन्नत्यिसत्कारानुगमाच्च मौलबलं भृतबलात्श्रेयः । नत्यियानन्तरं करषप्रि उत्थायविश्यं व भृतबलं शरेणीबलात्श्रेयः । जानपदं एकार्थ उपगतं तुल्यसङ्घर्ष्षामर्षसद्धिलाभं च श्रेणीबलं मत्रिबलात्र्रेयः । अपरमितिदेशकालें एकार्थ उपगमाचच मत्रिबलं अमत्रिबलात्र्रेयः । आर्याधष्ठितिं अमत्रिबलं अटवीबलात्श्रेयः । तद् उभयं वलोपार्थम् । अवलोपे व्यसने च ताभ्यों अहभियं स्यात् ।

${ }^{49}$ Kautilya, Arthaśāstra 7,2,4: यद्बलः सामन्तः तद्वशिष्टिबलं आश्रयेत् । तद्वशिष्टिबलाभावे तं एवाश्रतिः कोशदण्डभूमीनां अन्यतमेनास्य उपकर्तुं अदृष्टः प्रयतेत । महादोषो हविशिष्टिबलसमागमो राज्णां, अन्यत्रारविगृहीतात् । 
Der Herrscher, der diesen Charakteristiken gerecht werden will, darf sich keinerlei menschlichen Schwächen erlauben und muß sich beständig im Erreichen der notwendigen Herrschertugenden üben:

Durch die Ausstoßung der Gruppe von sechs Feinden soll demnach der Fürst den Sieg über die Sinne zuwege bringen, ebenso durch den Umgang mit den Fortgeschrittenen Einsicht, durch den Spionendienst Sehvermögen, durch kräftige Tätigkeit die Förderung des Neuerwerbs und des ruhigen Fortbesitzes (bei den Untertanen), durch Anbefehlung ihrer Pflichten die Aufrechterhaltung ihrer besonderen Lebensnorm, sittliche Zucht durch die Unterweisung in den Wissenschaften, Beliebtheit bei den Menschen dadurch, daß er ihnen irdische Vorteile verschafft, und durch das, was ihnen heilsam ist, gedeihliches Leben. ${ }^{50}$

Diese Tugenden sind offensichtlich, einmal mehr, ausschließlich erfolgsorientiert: Es geht nicht etwa darum, das Volk wie auch immer „besser“ zu machen und selbst in Zeiten der Krise zu Einsicht, Mäßigung und echter Frömmigkeit anzuhalten, selbst wenn dies gegen die Interessen des Staates verstoßen könnte, wie es in eher idealistisch ausgerichteten Texten zu lesen ist. ${ }^{51}$ Einzig der Erfolg heiligt die Mittel, und so versteigt sich das Arthaśästra sogar zu der Behauptung, daß die Ausübung des solchermaßen definierten Herrscheramtes der eigentliche „Gottesdienst“ des Herrschers sei und ihn somit gewissermaßen von den ansonsten geltenden religiösen Verpflichtungen freispreche; eine Sichtweise, welche freilich indirekt doch wieder fest im jenseitig motivierten Gesellschaftsbild der indischen Kultur verankert ist, ${ }^{52}$ wo die Erfüllung der Pflichten, die dem Menschen durch seine Geburt innerhalb einer bestimmten Gesellschaftsschicht zuwachsen, als höchste Aufgabe gilt, und das Aufgehen im gesellschaftsgerechten Tun zunehmend losgelöst vom eigentlichen Objekt des Tuns interpretiert wird:

Denn des Königs frommes Gelübde ist: kraftvolle Tätigkeit, sein Gottesdienst: die Anbefehlung dessen, was geschehen soll, seine Opferpriesterentlohnung: die Gleichmäßigkeit des Verhaltens und seine Weihe zu heiligem Werk: die feierliche Begießung mit Wasser. ${ }^{53}$

Hierbei zählen Ehre, Treue und Stolz offensichtlich nicht zu den erwünschten Herrschertugenden, denn während im Arthaśāstra Bhāradvāja und Visālāksha als Gewährsmänner einer jeweils religiös begründeten Unterwerfungs- bzw. Untergangsphilosophie zitiert (und offensichtlich implizit kritisiert) werden, ${ }^{54}$ gibt sich der Verfasser als Anhänger einer rein pragmatischen Anpassung an die Umstände:

${ }^{50}$ Kauțilya, Arthaśästra 1,7,1: तस्माद् अरषिड्वर्गत्यागेन इन्द्रयिजयं कुरवीत, वृद्धसम्योगेन प्रज़ां, चारेण चकष्: उत्थानेन योगक्षेमसाधनं, कार्यानुशासनेन स्वधर्मस्थापनं, वनियं वद्यिया उपदेशेन, लोकप्रयित्वं अर्थसम्योगेन वृत्तम्त् ।

${ }^{51}$ In dieser Hinsicht enthält der Text wohl sogar Spuren einer Abschwächung der amoralischen Grundhaltung zwischen der Daṇạaniti- und der Arthaśāstra-Redaktionsstufe; vgl. Mabbett 2010.

${ }_{52}$ Allg. hierzu Coomaraswamy 1942.

${ }^{33}$ Kautilya, Arthaśästra 1,19,9: राज्जो हव्रितं उत्थानं यज्जः कार्यानुशासनम् । दक्षणिा वृत्तसिाम्यं तु दीक्षा तस्याभषिचनम् ।I

${ }^{54}$ Bei Bhāradvāja handelt es sich um den halb-mythischen Verfasser des 6. Buchs des Rgveda, der auch im Mahäbhärata und im Rämāyaṇa wie auch in vielen anderen Schriften Erwähnung findet, und der als einer der (indischen) "Sieben Weisen" (Saptarșis) galt. Seine historische Einordnung ist infolge der komplexen Textgeschichte dieser über Jahrhunderte ausgeschriebenen und erweiterten Schriften nahezu unmöglich, ebenso wie der Versuch, die hier in Frage stehende Erwähnung als Beleg für die Existenz einer Bhāradvāja zugeschriebenen Staatsschrift zu sehen. Visālāksha scheint nur aus dem Arthaśāstra bekannt zu sein und ist 
„Von einem Stärkeren angegriffen, soll sich der Schwache in allen Fällen demütig beugen nach der Art des Rohrs. Denn vor Indra beugt sich, wer sich vor dem Stärkeren beugt.“ So Bhāradvāja. „Mit dem ganzen Aufgebot seiner Truppen soll er kämpfen. Denn die Tapferkeit tilgt das Unheil hinweg, und dies ist die ureigene Pflicht des Kriegers: Sieg oder Niederlage in der Schlacht." So Visālāksha. Nein, also Kauțilya. Wer sich in allen Fällen demütig beugt, der weilt hoffnungslos im Leben wie ein Küstenschaf. Und wer, nur mit einen kleinen Heere ausgerüstet, kämpft, der geht zugrunde wie einer, der ohne Schiff ins Meer hinausdringt. Aber unter den Schutz eines Königs, der mächtiger ist als jener, oder in eine uneinnehmbare Burg sich flüchtend, möge er sich betätigen. ${ }^{55}$

\subsection{Aineias Taktikos}

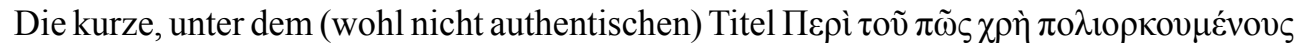

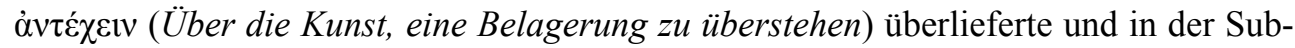
skription auch explizit mit dem Namen eines Aineias (Taktikos) ${ }^{56}$ verbundene Schrift ist die erste erhaltene militärwissenschaftliche Abhandlung der griechischen Literatur und sollte lange Zeit als Vorbild das neuentstandene Genre beherrschen. ${ }^{57} \mathrm{Ihr}$ Verfasser wird manchmal mit dem aus Xenophon bekannten gleichnamigen arkadischen General Aineias von Stymphalos verbunden, der um $367^{58}$ die politischen Verhältnisse in Sikyon neu zu ordnen versuchte und unter anderem die unter dem Tyrannen Euphron verbannten Bürger rückführen ließ; letzte Sicherheit läßt sich hier aber nicht gewinnen. Glaubt man den Anspielungen im Text selbst, schrieb Aineias Taktikos ebenfalls einige weite-

wohl in eine Reihe mit den zahlreichen anderen Gewährsmännern zu sein, die die Schrift zitiert, ohne doch nähere Anhaltspunkte für Datierung, Autorschaft und Inhalt (und sogar Historizität; vgl. Olivelle 2013, 6) zu liefern.

${ }_{55}$ Kauțilya, Arthaśästra 12,1,1-3: बलीयसाऽभयुक्तो दुर्बलः सर्वत्रानुप्रणतो वेतसधर्मा तषिठेत् । इन्द्रस्य हा स प्रणमता यो बलीयसो नमता इता भारद्वाजः । सर्वसन्दोहेन बलानां युध्येत, पराक्रमो ही व्यसनं अपहन्ता। स्वधर्मश्च एष कषत्रयिस्य, युद्धे जयः पराजयो वा' इता विशालाकषः । न इतकिट्यिल्यः । सर्वत्रानुप्रणतः कुलैडक इव नरिशो जीवति वसता । युध्यमानश्चाल्पसैन्यः समुद्रं इवाप्त्वोड वगाहमानः सीदती तद्वशिष्टिं तु राजानं आश्रति दुर्गं अवषिह्यं वा चेष्टेत।

${ }^{56}$ Der Name des Verfassers wird auch in der Schrift selbst erwähnt: vgl. 31,8. Allg. zu Aineias Taktikos vgl. Hug 1877; Lange 1879; Schwartz 1894; Hudson-Williams 1904; Hunter - Handford 1927; Marinovich 1962; Bengtson 1962; Celato 1968a und 1968b; Anderson 1970; Tuplin 1976; Lehmann 1980; Brown 1981; David 1986; Urban 1986; Lehmann 1989; Winterling 1991; Vela Tejada 1991; Vela Tejada 1992; Whitehead 1992; Vela Tejada 1993; Whitehead 2002; Günther 2014; Wu 2016.

${ }^{57}$ So ließ König Pyrrhos den Kineas einen Auszug aus Aineias' Schrift anfertigen (Ael. Takt. 1,2), und sein Einfluß läßt sich von Polybios über Onasander, Claudius Aelianus, Polyainos und Philon von Byzanz bis hin zu Iulius Africanus nachweisen. Das heute erhaltene Werk entstammt allerdings nur einem einzigen Codex (Laurentianus 55,4), auf dem alle weiteren Handschriften gründen, und der selbst auf eine von Konstantinos VII. Porphyrogennitos angefertigte Sammlung zurückgeht.

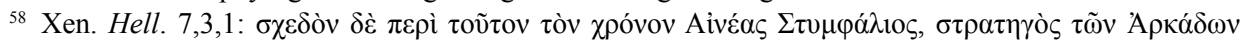

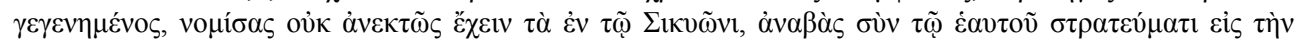

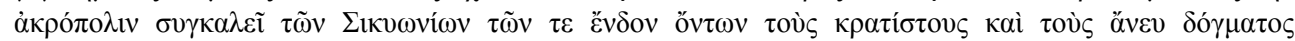
$\dot{\varepsilon} \kappa \pi \varepsilon \pi \tau \omega \kappa o ́ \tau \alpha \varsigma ~ \mu \varepsilon \tau \varepsilon \pi \varepsilon ́ \mu \pi \varepsilon \tau o$. (Zu dieser Zeit rief Aeneas von Stymphalon, der zum Heerführer der Arkader geworden war, der dachte, daß die Verhältnisse in Sikyon nicht mehr zu ertragen seien, und der mit seinen Soldaten zur Akropolis heraufgegangen war, die Mächtigen unter den Sikyoniern, die in der Stadt waren, zusammen, und rief diejenigen, die ohne einen Volksbeschluß vertrieben worden waren, wieder zurück.) Siehe zu Euphron auch Xen. Hell. 7,1,44-46 und Diod. 15,70,3. Die umfassendste Argumentation für eine solche Identifizierung findet sich bei Hug 1877; dagegen bei Lange 1879 und Hudson-Williams 1904. 
re militärwissenschaftliche Abhandlungen, die allerdings alle verloren sind; ${ }^{59}$ selbst die erhaltene Schrift bricht vorzeitig ab und läßt kaum Rückschlüsse auf das Fehlende zu.

Diese zeitliche Verortung ist nicht unwesentlich für das Verständnis des Textes: auf die weitgehend unangefochtene Herrschaft der aristokratischen Oberschicht und den Höhepunkt griechischer „Einheit“ gegenüber dem achaimenidischen Eroberer in den Perserkriegen folgte im 5. Jh. ein Zeit erbitterter zwischenstaatlicher Konflikte zwischen den großen spartanischen und attischen Bündnissystemen, welche in die schrecklichen Verwüstungen des Peloponnesischen Krieges mündete, bald darauf aber unter anderen, stetig wechselnden Konstellationen weiter fortgesetzt wurde, bis es schließlich unter makedonischem Druck zu einer erneuten Vereinigung Griechenlands in Form des Korinthischen Bundes kam. ${ }^{60}$ Dieses Zeitalter, das künstlerisch als klassische Epoche gewürdigt und politisch von einem fast bruchlosen Kriegszustand geprägt wurde, hatte nicht nur Auswirkungen auf die stets verfeinerte Militärtechnik, ${ }^{61}$ sondern auch die Theorie zwischenstaatlicher Beziehungen, ${ }^{62}$ und es ist kein Wunder, daß es gerade in jener Zeit war, daß das Werk des Aineias Taktikos entstand.

Aineias Taktikos' poliorketische Schrift ist nicht nur ein wichtiger, ja vielleicht der wichtigste Zeitzeuge der militärtheoretischen Geschichte des spät-klassischen Zeitalters und eine wertvolle Quelle für unsere Kenntnis des „dritten Griechenlands“ jenseits von Athen und Sparta. ${ }^{63}$ Er ist auch eines der wichtigsten Zeugnisse für die Entstehung eines pragmatisch und realistisch ausgerichteten griechischen Staatsdenkens, denn wenn dessen Ursprünge auch sicherlich im Kreise der sophistischen Lehre des 5. Jh.s zu finden sind, deren Trümmer sich aus der polemischen Verzerrung bei Platon nur ungenügend rekonstruieren lassen, ${ }^{64}$ scheinen jene politischen Lehrer wesentlich durch mündliche, nicht schriftliche Unterweisung gewirkt zu haben, ${ }^{65}$ so daß, von einigen Spuren in den Historikern abgesehen (man denke hier v.a. an Thukydides' Melierdialog, aber auch an Überlegungen bei Xenophon), Aineias Taktikos unsere erste greifbare Spur eines solchen praktisch-rationalistischen Staatsdenkens ist. Denn selbst wenn die Schrift auch wesentlich militärwissenschaftlich ausgerichtet ist, transportiert sie doch bereits ebenso durch ihre recht apolitische Grundhaltung wie auch ihren Verzicht auf normative und idealistische Verfahrensweisen zugunsten reiner, durch zahlreiche Fallbeispiele untermauerte Praxisempfehlungen eine Grundaussage, welche etwa dem politisch-philosophischen Werk eines Platon diametral entgegensteht.

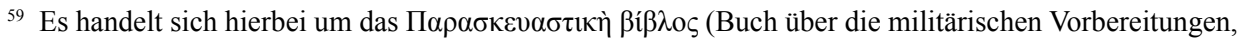

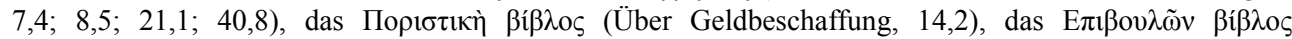

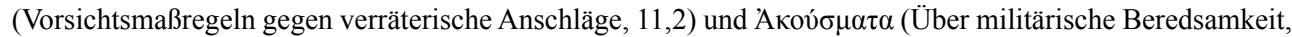
38,5). Zusätzlich kündigt der Autor ein Werk über Feldzüge an $(21,2)$. Auch ein vielleicht Aineias Taktikos zuzuschreibendes Buch über „Taktik“ ist erwähnt (Ael. Takt. 3,4), aber umstritten.

${ }^{60}$ Allg. zum politischen Kontext mit besonderem Bezug auf Aineias s. Schulz 1999.

${ }^{61}$ Zum militärischen Kontext s. Anderson 1970; Hanson 1989 und 1991; Tejada 2004.

62 Mosley 1973.

63 Vgl. Gehrke 1986; vgl. auch Lehmann 1989.

${ }^{64}$ S. pars pro toto Plat. Pol. 344c.

65 S. hierzu auch z.B. den in Xen. Apomn. 3,1,1ff. erwähnten militärtechnischen Vortrag des Sophisten Dionysodoros (von Chios), der auch zusammen mit seinem Bruder Euthydemos bei Plat. Euth. 273c als Militärspezialist Erwähnung findet. Die früheste Verschriftlichung einer solchen Unterweisung (hier über das Reiten) aus der Feder des Simon von Athen ist bei Xen. Hipp. 1,1; 1,3; 11,6 erwähnt. S. allg. Vela Tejada 1993. 
Dies zeigt bereits die Ausrichtung und Struktur des wohl in den späten 350er Jahren entstandenen Werks. ${ }^{66}$ Die Abhandlung ist offensichtlich an militärtechnische Laien gerichtet und beweist hiermit offensichtlich den hohen Grad der potentiellen Militarisierung auch der zivilen Polis-Elite, wenn freilich nicht unterschätzt werden darf, daß der intensive Militärdienst, den die freien Bürger abzuleisten hatten, eine erheblich größere Vertrautheit mit dem Waffenhandwerk bewirkte, als dies etwa in anderen Gesellschaften des Altertums der Fall war, die eher auf das Söldnerwesen oder eine Berufsarmee vertrauten. Gegliedert - wenn man das bei einer offensichtlich so chaotischen bzw. rein assoziativen Anordnung

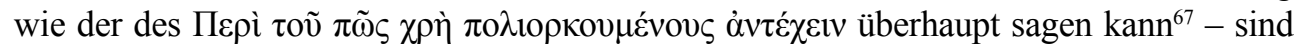
die (erhaltenen) Teile des Werks in sechs Abschnitte: Auswahl und Stationierung von Truppen (1-10.24), Aufrechterhaltung der öffentlichen Moral (10.25-14), Zurückschlagen von unerwarteten Einfällen (15,1-16,15), Abwehr feindlicher (oder revolutionärer) Angriffe auf die Stadttore bei Prozessionen (16,16-20), Bewachung der Mauer und Unterbindung von Waffenschmuggel (22-31) und schließlich Abwehr feindlicher Angriffe auf die Befestigungsanlagen (32-40), wobei bemerkenswert ist, daß der Autor inneren Umstürzen generell mehr Gewicht zumißt als äußeren Angriffen, ${ }^{68}$ und fast alle seiner Ratschläge mit umfangreichen exempla aus der jüngeren Geschichte Griechenlands untermauert, die in vielem den Einfluß Herodots und Thukydides' verraten.

In Aineias Taktikos' Schrift über die Belagerung von Städten finden wir zahlreiche Elemente, die uns an das erinnern, was wir auch im chinesischen und indischen Kontext bereits besprochen haben. Wichtigstes Ziel der Schrift ist es, daß die belagerte Stadt um jeden Preis so lange wie möglich verteidigt wird bzw. ihre etablierte politische Ordnung gegen alle inneren Widerstände beibehält; alles andere hat sich diesem selbstgewählten argumentativen Ziel unterzuordnen. Hierbei ist beachtlich, daß die Absolutheit dieser selbstbestimmten Absicht deutlich mit dem Pragmatismus und dem Relativismus der aufgezählten Mitteln kontrastiert, was einmal mehr nahelegt, daß es dem Autor eben nicht um die Einordnung der Fragestellung in ein übergeordnetes, moralisches, rechtliches oder religiöses Konzept geht, sondern um die sachliche Lösung einer abstrakten Aufgabe:

Diejenigen also, die um so viele und so große Güter streiten wollen, dürfen es an keinerlei Vorbereitung und Zuversicht fehlen lassen, sondern müssen zahlreiche und vielfältige Arbeiten vorab versehen, damit sie jedenfalls nicht durch ihr eigenes Tun zu scheitern scheinen. Wenn also ein Unglück geschieht, können wenigstens die übrig Bleibenden das Bewahrte später einmal zu seiner früheren Art wieder herstellen - so, wie manche der Griechen, die zum Äußersten gekommen waren, sich wieder erhoben haben. ${ }^{69}$

${ }^{66}$ Die Aufstellung eines terminus post quem ergibt sich daraus, daß die jüngsten historischen Anspielungen in die Zeit zwischen 359 und 355 gehören $(4,1 ; 11,3 ; 28,6 ; 31,31$; hierzu Whitehead 2002 ad loc.). Der terminus ante quem wird oft aus 31,24 gewonnen, wo Aineias den 346 abgeschafften (Apollod. Epit. 23,9 und Tim. Taur. FGrHist 566 F 146b) lokrischen Jungfrauen-Tribut an Troia/Ilion als immer noch gültig bezeichnet.

${ }^{67}$ Allg. zur Schwierigkeit der Struktur des Werkes Whitehead 2002, 17-21.

68 Hierzu besonders Winterling 1991.

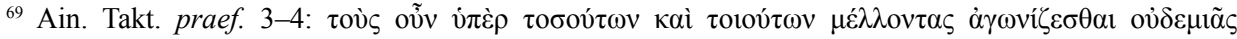

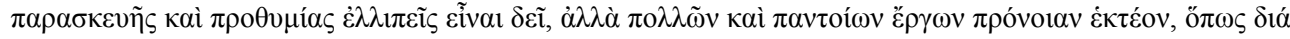

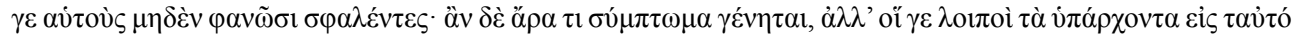

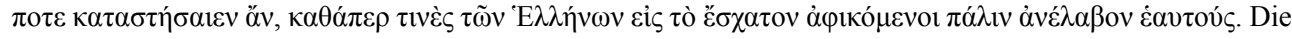
deutsche Übersetzung folgt der von Brodersen 2017. 
Der Text ist demgemäß zutiefst unpolitisch, und wenn gelegentlich doch Sympathien und Antipathien durchzuschimmern scheinen, legt Aineias sich doch offensichtlich nicht auf eine eigene politische Sichtweise fest, sondern gibt sich rein fachwissenschaftlich und relativiert durch die Wahl der angeführten exempla.$^{70}$ Dem entspricht ein eher desillusioniertes Menschenbild, und wenn man auch Ausführungen zu eher religiösen Fragen vergebens suchen wird ${ }^{71}$ - vielleicht befanden sie sich in den verlorenen Teilen des Traktates -, zeigen doch die Bemerkungen zu eher innenpolitischen Fragen, daß Aineias ausschließlich Nützlichkeitserwägungen, nicht aber übergeordnete Rechtsfragen in den Mittelpunkt seiner Überlegungen stellen will. So lesen wir gar, daß die Auswahl der Offiziere nicht nur nach ihrer fachlichen Befähigung zu erfolgen hat, sondern auch nach dem Gesichtspunkt ihrer rein persönlich motivierten Furcht vor einem möglichen Regimewechsel (egal, in welcher Richtung dieser nun ausschlagen würde):

Diese müssen gutgesinnt und mit der bestehenden Politik zufrieden sein: etwas Großes angesichts der Verschwörungen der anderen steht ja in etwas so Gemeinsamem bereit - wie eine Akropolis; es soll ein Gegenstand der Furcht für die sein, die in der Stadt etwas Gegenteiliges wollen. Der Anführer und Verwalter von ihnen soll im Allgemeinen umsichtig und energisch sein und jemand, der die größten Gefahren bei einem Umschwung riskiert. ${ }^{72}$

Aineias warnt auch eindringlich vor den Metöken, die in der bedrohten Stadt leben, als vor möglichen Feinden und Spionen, welche gewissermaßen nur auf eine Gelegenheit warten, die Stadt auszukundschaften oder gar die innere Ordnung zu ihren Gunsten umzustürzen. ${ }^{73}$ So sollen diese selbst von Militärparaden ferngehalten werden, die es ihnen ermöglichen könnten, die tatsächliche Stärke des Heeres der Stadt zu ermessen und diese Information weiterzugeben oder zur Ausarbeitung potentiell feindlicher Pläne zu nutzen:

Häufige Musterungen haben stattzufinden und die in der Stadt lebenden Fremden haben sich während dieser Zeit an einem bestimmten Ort einzufinden oder im Haus zu bleiben; wenn sich aber einer woanders sehen läßt, ist er als ein Übeltäter zu bestrafen. ${ }^{74}$

Gleichzeitig gilt es auch, beständig Jagd auf mögliche Verräter in den eigenen Reihen $\mathrm{zu}$ machen und ferner besonders auf die Überprüfung der Identitätsnachweise zu

${ }^{70}$ In diesem Kontext sei allerdings verwiesen auf von Pöhlmann (1925, I: 336-338) und Marinovich (1962, non vidi), welche beide, wenn auch aus unterschiedlicher ideologischer Perspektive, Aineias Taktikos als anti-demokratischen, pro-oligarchischen (bzw. pro-aristokratischen) Techniker betrachteten. Dagegen dann Hunter - Handford 1927, 233, welche ihn vielmehr aus einer pro-demokratischen Perspektive lasen.

${ }^{71}$ Freilich erwähnt auch Aineias häufig Prozessionen, Altäre oder Götterschwüre als gegebene gesellschaftliche Größen; im Gegensatz zu den anderen hier untersuchten Schriften enthält er sich jedoch jeglicher Anweisungen, wie die kultischen Interaktionen mit der göttlichen Sphäre im Interesse der oder des Herrschenden manipuliert werden können.

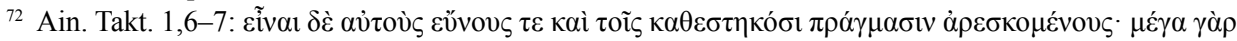

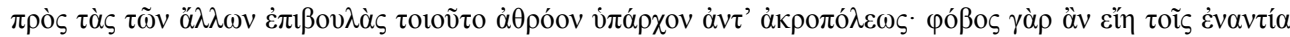

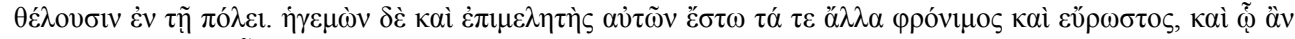

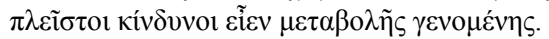

${ }^{73}$ Zum Fremdenbild des Aineias vgl. Whitehead 1992. Hier sind wohl Fremde gemeint, die dauerhaft in der Stadt seßhaft waren, und die z.B. in Athen (Whitehead 1977) und auch anderen Städten wie Megalopolis Teil des Heeres waren (Diod. 18,70,1).

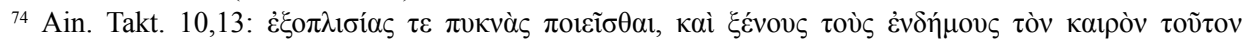

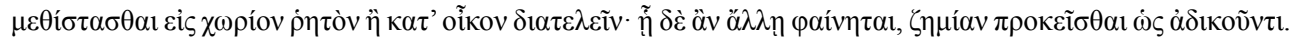


achten: ${ }^{75}$ eine Erfahrung, die sich aus den nahezu ungezählten Präzedenzfällen der klassischen griechischen Geschichte speist, wo aus dem Exil zurückgekehrte Bürger die Macht usurpierten. Aineias fordert daher von seinem idealen Festungskommandanten, die Bürger möglichst zur Denunziation aufzufordern, um jeden inneren Widerstand gegen die herrschende Partei auszuschalten, ohne doch darauf zu achten, wie sehr ein solches inneres Klima meist gerade jene Verschwörung fördert, denen vorgebeugt werden soll:

Für den Fall, daß jemand einen Verschwörer gegen die Stadt angibt oder einen Übertreter der vorher beschriebenen Befehle anzeigt, ist für diesen Geld festzulegen und dieser Betrag öffentlich auf der Agora oder bei einem Altar oder in einem Heiligtum bekannt zu machen, damit man umso bereitwilliger etwas von dem vorhin Beschriebenen anzuzeigen wagt. ${ }^{76}$

Der Krieg als Ausnahmesituation (wenn auch offensichtlich ist, daß die Stadt sich letztlich permanent in einem mehr oder weniger latenten Kriegszustand nicht nur mit den umliegenden Nachbarn, sondern auch mit der eigenen innenpolitischen Opposition befindet) ${ }^{77}$ zwingt dabei zu allen Maßnahmen, die der Sicherung des Überlebens dienen können, auch wenn sie auf eine völlige Umkehrung der gesellschaftlichen Ordnung hinauslaufen könnten. So rät Aineias auf der einen Seite zu einer Art „Kriegssozialismus“ zur Stärkung des inneren Zusammenhaltes:

Die Masse der Bürger muß man aber einstweilen ganz und gar einträchtig machen, teils durch andere Maßnahmen, teils indem man den Schuldnern die Zinslast erleichtert oder ganz erläßt, bei großer Gefahr auch einen Teil der Schulden oder auch alle, wenn es nötig ist, da Menschen dieser Art oft gerade die gefährlichsten Elemente sind. Auch muß man denjenigen, die am Notwendigen Mangel leiden, bessere Versorgung gewähren. ${ }^{78}$

Auf der anderen Seite aber erfordert der Krieg nicht nur einen harschen Umgang mit möglicherweise feindlichen Fremden, sondern auch mit mißliebigen eigenen Bürgern, wobei hier nicht nur jene in Betracht kommen, die sich tatsächlich durch eine offen staatsfeindliche Haltung hervorgetan haben, sondern auch jene, die nur als mögliche Dissidenten und Unruhestifter bekannt sind. Aineias geht hier sogar soweit, dem Befehlshaber bzw. der die Stadt beherrschenden Partei anzuraten, potentielle innere Feinde unter unauffälligen Gründen längere Zeit aus der Stadt zu entfernen, und es bedarf nicht viel Einbildungskraft, den von Aineias aufgezählten, „diplomatischen“ Herangehensweise einige weitere, weniger friedliche hinzuzuzählen:

Danach ist für die übrigen Anordnungen Sorge zu tragen. Zuerst muß man zusehen, ob die Bürger einträchtig sind, da dies bei einer Belagerung das höchste Gut ist, wenn sie es nicht sind, muß man einige von denen, die mit dem Bestehenden unzufrieden sind $\langle\ldots>$ und namentlich die Anführer

${ }^{75}$ Ain. Takt. 10,8; es geht hier wohl um die aus Aristoph. Orn. 1212-1215 bekannten Symbola.

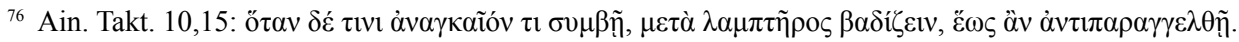

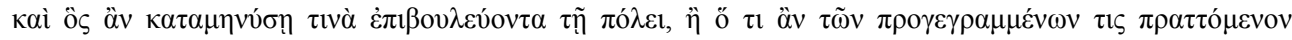

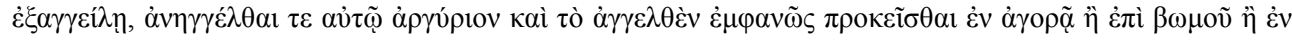

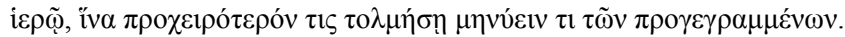

77 Dazu auch Plat. Nomoi 626a.

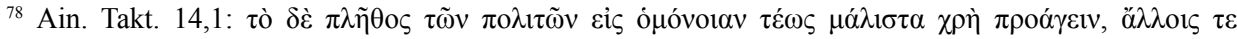

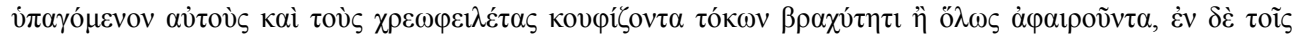

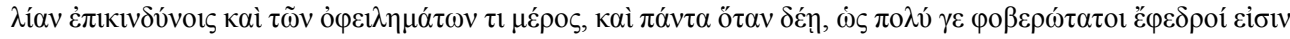

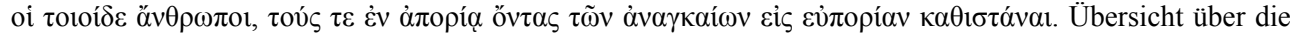
umfassende Literatur zu dieser Stelle bei Whitehead 2002, 137. 
und diejenigen, die in der Stadt einen Handstreich anstiften könnten, auf unverdächtige Weise entfernen, indem man sie unter einem wohlklingenden Vorwand als Gesandte und in anderen öffentlichen Aufträgen irgendwohin schickt. ${ }^{79}$

Unter diesen Umständen ist kaum erstaunlich, daß ein ganzes Kapitel (11) verschiedensten Formen von Verrat und Betrug gewidmet ist; eine sich nicht nur aus dem unvermeidlichen „Lagerkoller“ der belagerten Stadt ergebende Konsequenz, sondern auch eine, die unmittelbar aus dem Menschenbild des Aineias und seiner Zeit resultiert und durchaus mit der faktischen Wirklichkeit beständiger ziviler stasis, also bürgerkriegsähnlicher Tendenzen, übereinstimmt. ${ }^{80}$

Auch vor den eigenen Bundesgenossen gilt es daher, sich in acht zu nehmen, und die Verläßlichkeit ihrer Hilfstruppen wird beständig in Frage gestellt, was wohl sehr aussagekräftige Rückschlüsse auf die generelle Einschätzung zwischenstaatlicher Beziehungen erlaubt:

Wenn Bundesgenossen in die Stadt $\langle\ldots>$, dürfen niemals die Bundesgenossen sich alle zusammen aufhalten, sondern müssen ebenfalls getrennt werden aus denselben, vorhin benannten Gründen. Ebenso müssen auch, wenn man in Verbindung mit Söldnern etwas unternehmen will, diejenigen Bürger, die jene in dienst nehmen, an Zahl und stärke die Söldner übertreffen; anderenfalls werden sie selbst und ihre Stadt in die Hände jener fallen. ${ }^{81}$

Aineias erwähnt leider an keiner Stelle seines Werks die erwünschte prototypische Persönlichkeit des anonymen Feldherrn oder Festungskommandanten selber, an den die Schrift doch offensichtlich gerichtet ist; ${ }^{82}$ wenn der Text also auch durchaus Anklänge an zeitgenössische Fürstenspiegel zeigt (man denke hier nur an Xenophons Kyroupaidia oder Hieron), so bleibt sie doch ganz auf das Handeln, nicht das Sein (oder Scheinen) des Machthabers beschränkt. Sollten diesbezügliche Ausführungen sich nicht etwa in den nunmehr verlorenen Partien der Schrift befunden haben, wäre dieses Fehlen einer Beschreibung der optimalen Befähigungen des Kommandanten in bereits mehr als einer Hinsicht interessant. Zum einen nämlich zeigt gerade die Abwesenheit solcher Ausführungen den Wunsch, der Schrift jegliche Auseinandersetzung mit Fragen von Moral, Charakter und Gerechtigkeitssinn zu nehmen, der sich unweigerlich eingestellt hätte, und entspricht somit der rein pragmatischen Zielsetzung, der es nicht um Menschenerziehung geht, sondern um Hilfestellung in schwieriger Lage, ganz außerhalb der jeweiligen Befähigung des Adressaten. Zum anderen ist klar, daß das Feldherrenamt und somit die oberste Befehlsgewalt als wesentlich monokratisch empfunden wird, lesen wir doch nirgendwo etwa von einer notwendigen Abstimmung des Entscheidungsträgers mit

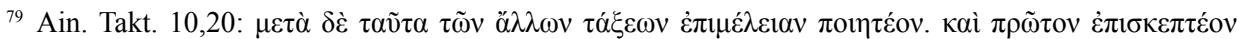

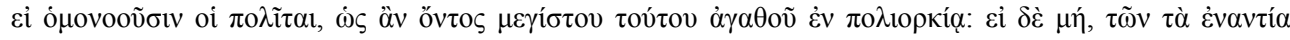

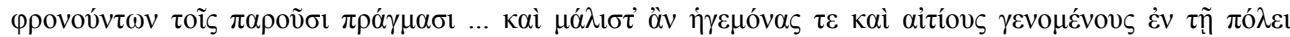

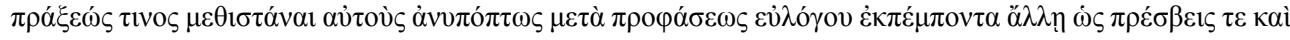

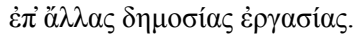

${ }^{80}$ Hierzu Lintott 1982; Gehrke 1985 und bes. Urban 1986.

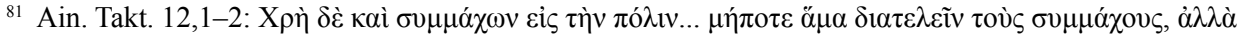

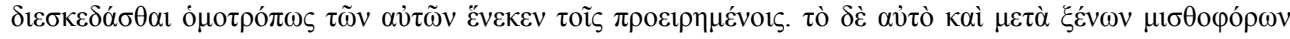

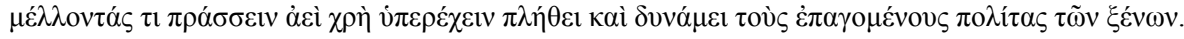

${ }^{82}$ Zur Frage, inwieweit die Schrift an einen spezifischen Adressaten oder Adressatenkreis gewidmet ist (wie etwa Xenophons Poroi), oder als rein theoretische Abhandlung entstand, vgl. Whitehead 2002, 34-42. 
etwaigen Kollegen, die sich aus einer demokratischen oder oligarchisch-aristokratischen Staatsform ergeben könnte, während dem Feldherrn durchaus zivile Kompetenzen wie das Absenden von Botschaften oder die Neuregelung der Schulden zugeschrieben werden. Freilich ist das Werk keineswegs als Plädoyer für eine Monarchie zu lesen; die Unterstellung der Alleinverantwortlichkeit des Befehlshabers und seines oft recht freien Umgangs mit den ihm zur Verfügung stehenden Machtmitteln verrät allerdings schon durchaus den geistig-politischen Kontext der Jüngeren Tyrannis. ${ }^{83}$

\subsection{Machiavelli}

Der Il Principe des Niccolò Machiavelli ${ }^{84}$ (1469-1527) ist bekannt genug, um es hier bei einer sehr gedrängten Zusammenfassung belassen zu können. Auch bei dieser Schrift, seit 1513 als eine Art Epitome aus den umfassenderen Discorsi sopra la prima Deca di Tito Livio entstanden und wie diese erst posthum veröffentlicht, handelt es sich um den ersten pragmatischen und bewußt von jeglicher Form moralischer Rücksicht befreiten „Fürstenspiegel“" der europäischen Geschichte, was den Principe grundsätzlich von den bisherigen Ansätzen unterscheidet. 1469 in Florenz ${ }^{85}$ als Sohn des Juristen Bernardo Machiavelli geboren, der 1476 schon einen toponymischen Index des livianischen $A b$ Urbe condita verfaßt hatte, genoß Niccolò Machiavelli eine solide lateinische Bildung und wurde zum Zeuge der italienischen Kriege, während derer der neue französische Territorialstaat und das Heilige Römische Reich sich um die Hegemonie über Italien stritten und die ganze Machtlosigkeit der italienischen Kleinstaaten wie auch des Kirchenstaates offenbarten. Nach dem Fall Piero de' Medicis und der kurzfristigen Theokratie Savonarolas wurde Machiavelli 1498 zum Sekretär der Zweiten Kanzlei und somit mitverantwortlich für die Ausgestaltung der auswärtigen Beziehungen und militärischen Organisation Florenz' und unternahm mehrere längere Auslandsreisen. Nach dem Höhepunkt seiner Karriere, als er 1509 die Rückeroberung Pisas überwachte, folgte 1512 mit der Rückkehr der Medicis sein Fall und das Exil, das er zu einer intensiven literarischen Tätigkeit nutzte, wobei neben den Discorsi und dem Principe in unserem Kontext v.a. das Dell'arte della Guerra $(1521)^{86}$ und die Istorie fiorentine (1525) hervorzuheben sind. 1525 gelang die Rückkehr in die Politik und die Übernahme des Ausbaus der Befestigungen Florenz', auf die 1527 nach dem Sacco di Roma und dem erneuten Fall der Medici der endgültige Rückzug und der rasche Tod in der Verarmung folgten.

Im Gegensatz zu seinen Vorgängern, die entweder vom Genre der politischen Utopie ausgingen, wie Palmieri und Patrizi, oder vom antiken Erbe des Fürstenspiegels als moralisierender und überzeitlicher Idealdarstellung, wie bei Poggio, Bracciolini und

83 Allg. hierzu Frolov 1974.

${ }^{84}$ Allg. zur Biographie Machiavellis: Ridolfi 1972; Garin 1993; Viroli 1998; Hoeges 2000; Unger 2011; Weibel 2012; Celenza 2015.

85 Zum Florentiner Kontext s. Baron 1955; Münkler 1982; Godman 1998.

${ }^{86}$ Im Rahmen dieser Untersuchung wurde allerdings der Principe der Arte della Guerra bevorzugt, da letzte Schrift fast gänzlich im geistigen Horizont römischer Militärkunst verortet ist und nur gelegentlich Einblicke in übergeordnete staatstheoretische Überlegungen erlaubt. 
Ficino, ${ }^{87}$ gehen Machiavellis staatstheoretische Arbeiten von konkreten exempla und der sich hieraus ergebenden induktiven Methode aus, welche in starkem Kontrast zur bisherigen, eher deduktiven Herangehensweise steht. Im Mittelpunkt steht dementsprechend, v.a. im Principe, kein von religiösen, rechtlichen oder sittlichen Vorstellungen abgeleitetes herrscherliches oder gesellschaftliches Idealbild, sondern vielmehr die Überlegung, mit welchen Mitteln Herrschaft erlangt, gewahrt, verteidigt und vermehrt werden könne. ${ }^{88}$ Die jeweiligen gesellschaftlichen und geistlichen Rahmenvorstellungen werden zwar als gegeben hingenommen und in ihrer Berechtigung kaum weiter diskutiert, werden aber als bloße Mittel zum Zweck bzw. als Hindernisse auf dem Weg zur Macht angesprochen.

Dieser Pragmatismus verdankt sich nicht nur dem neuentdeckten antiken Erbe, dessen Erfahrungen, hierbei vor allem aus Titus Livius entwickelt, ${ }^{89}$ Machiavelli auf Schritt und Tritt in zeitgenössisch relevante politische Maximen umzusetzen sucht; auch der historische Kontext war einem solchen Paradigmenwechsel überaus förderlich. Denn wie bereits die anderen hier vorgestellten Autoren lebte auch Machiavelli mitten in einer ereignisreichen und grundsätzlichen Umbruchszeit. ${ }^{90}$ Zum einen erlebte der florentinische Autor den Zusammenbruch des alten, universalistischen Weltbildes, welches durch das Heilige Römische Reich deutscher Nation verkörpert und durch den letzten Aufschwung des Habsburgischen Universalismus Karls V. wie auch durch den übernationalen Einfluß des Papsttums symbolisiert wurde; zum anderen wurde er zum Zeuge des Aufstiegs nicht nur der neuen Territorialstaaten wie etwa Frankreich oder Spanien, sondern auch eines neuen Menschenbilds, das zum einen etwa durch die Renaissance, zum anderen durch den Protestantismus versinnbildlicht wurde. ${ }^{91}$

Diese Ausgangsbedingungen erklären dann auch die komplexe Struktur des kurzen Werkes, das in 26 Kapitel unterteilt ist. Die ersten 8 Kapitel sind der Frage nach der Erwerbung der Herrschaft gewidmet, die Kapitel 9-11 den Arten der Herrschaft, Kapitel 12-14 dem Heerwesen, Kapitel 15-19 den nötigen persönlichen Eigenschaften und Kenntnissen des Fürsten, Kapitel 20-25 Einzelfragen, die vom Festungsbau über Verwaltung und Umgang mit Schmeichlern bis hin zur Rolle des Glücks reichen, und Kapitel 26 schließlich dem Aufruf, sich Italiens zu bemächtigen und es von den Barbaren zu befreien (Exhortatio ad capessendam Italiam in libertatemque a barbaris vindicandam). Dies macht deutlich, daß im Mittelpunkt von Machiavellis Werk die reine Optimierung der Herrschaft im Zentrum steht, und so schreibt er denn auch explizit:

Indem ich also die bloßen Vorstellungen über den Fürsten beiseite lasse und nur von seiner Wirklichkeit spreche... ${ }^{92}$

${ }^{87} \mathrm{Zu}$ Machiavellis Vorbildern vgl. Gilbert 1938; Horkheimer 1971; Godman 1998.

${ }^{88}$ Zum Denken Machiavellis s. Strauss 1958; Chabod 1965; Gennaro 1965; Buck 1985; Bock - Skinner - Viroli 1990; Najemy 2010; Jurdjevic 2014; Rossi Leidi 2015.

${ }^{89} \mathrm{Zu}$ Machiavelli und Livius vgl. jetzt allg. Engels $2017 \mathrm{~b}$.

${ }^{90}$ Zum Geist der Renaissance s. Hale 1961; Burckhardt 1860/1976; Capponi 2010; Oppenheimer 2011; Connell 2015.

${ }^{91}$ Allg. zum historischen Kontext Machiavellis vgl. von Albertini 1955; Hale 1961; Pullan 1973; Hay 1977; Goez 1984; Ruggiero 2015.

92 Machiavelli, Principe 15: Lasciando, adunque, indrieto le cose circa uno principe imaginate, e discorrendo quelle che sono vere [...]. Machiavellis Principe soll im folgenden nach der Edition und Übersetzung von Rippel 1986 zitiert werden. 
Auch das Ziel des idealen Fürsten ist offensichtlich: Machiavelli geht es nicht nur um das bloße Überleben des Herrschers und seines Staates, sondern auch der sukzessiven Ausdehnung seiner Macht, an deren Ende dann der Traum des florentinischen Denkers stehen soll: Die Vereinigung Italiens und, hieraus implizit folgend, die Wiedererrichtung zumindest der Würde des ganz als itali(eni)sche Schöpfung verstandenen römischen Reiches. $^{93}$

Man sollte also diese Gelegenheit nicht vorübergehen lassen, damit Italien nach so langer Zeit seinen Retter erblickt. Ich kann es nicht ausdrücken, mit welcher Liebe er in all den Gebieten empfangen würde, die unter der Überflutung durch die Fremden gelitten haben; mit welchem Durst nach Rache, mit welch unverbrüchlicher Treue, mit welcher Ergebenheit und welchen Tränen. Welche Türen würden sich ihm verschließen? Wer im Volk würde ihm den Gehorsam verweigern? Wessen Neid würde sich ihm entgegenstellen? Welcher Italiener würde ihm die Huldigung versagen? Jeden widert die Herrschaft der Barbaren an. ${ }^{94}$

Daher sind auch grundlegende Werte wie Religion, Humanität oder Treue nur so lange wünschenswert, wie sie dem Machterhalt dienen, und dürfen vom idealen Fürsten allein unter diesen Gesichtspunkten betrachtet werden. Machiavelli ist sich dabei der besonderen Bedeutung der Frömmigkeit durchaus bewußt, betrachtet sie aber nicht als einen absoluten Wert an sich, was etwa das seelische Wohlbefinden des Fürsten betrifft, sondern nur als einen historisch gegebenen gesellschaftlichen Faktor, den es eben genauso zu berücksichtigen gilt, wie Geographie oder strategische Nachbarschaftsverhältnisse: ${ }^{95}$

Ein Fürst muß also sehr darauf achten, daß nie ein Wort über seine Lippen kommt, das nicht von den vorgenannten fünf Eigenschaften geprägt ist, und daß er, wenn man ihn sieht und hört, ganz von Milde, Treue, Aufrichtigkeit, Menschlichkeit und Frömmigkeit erfüllt scheint. Und es gibt keine Eigenschaft, deren Besitz vorzutäuschen, notwendiger ist, als die letztgenannte. ${ }^{96}$

Auch Machiavellis Menschenbild ist also wesentlich von Mißtrauen, Berechnung und Manipulation geprägt. Dementsprechend häufig setzt er sich auch mit Fragen auseinander, wie der Fürst möglichen Verschwörungen aus dem Weg gehen kann, und wenn er auch (seltsamerweise) der Spionage zumindest im Principe keine eigenständige Betrachtung widmet, ${ }^{97}$ wird doch klar, daß der ideale Herrscher keinem Menschen wirkliches Vertrauen schenken darf.

Dies hat auch auf die Sichtweise zwischenstaatlicher Beziehungen großen Einfluß. Machiavelli fordert ja bereits von seinem ,eigenen“ Fürsten, daß er sich nur so lange an Verträge halten solle, wie dies vorteilhaft für ihn sei:

${ }^{93} \mathrm{Zu}$ den „letzten Zielen“ Machiavellis vgl. Hörnqvist 2004; Landon 2005.

${ }^{94}$ Machiavelli, Principe 26: Non si deve adunque lasciar passare questa occasione, acciocchè la Italia vegga dopo tanto tempo apparire un suo redentore. Nè posso esprimere con quale amore ei fussi ricevuto in tutte quelle provincie che hanno patito per queste illuvioni esterne, con qual sete di vendetta, con che ostinata fede, con che pietà, con che lacrime. Quali porte se gli serrerebbono? Quali popoli li negherebbono la obbidienza? Quale invidia se gli opporrebbe? Quale Italiano gli negherebbe l'ossequio? Ad ognuno puzza questo barbaro dominio.

${ }_{95} \mathrm{Zu}$ Machiavellis Haltung gegenüber dem Christentum vgl. Sullivan 1996; Viroli 2005; Parsons 2016.

${ }_{96}$ Machiavelli, Principe 18: Debbe, adunque, avere un Principe gran cura che non gli esca mai di bocca una cosa che non sia piena delle soprascritte cinque qualità; e paia, a vederlo e udirlo, tutto pietà, tutto fede, tutto integrità, tutto umanità, tutto religione. E non è cosa più necessaria a parere di avere che questa ultima qualità.

${ }^{97}$ Dafür erscheint das Thema etwas ausführlicher in Dell'arte della guerra; v.a. in Buch 6. 
Ein kluger Herrscher kann und darf daher sein Wort nicht halten, wenn ihm dies zum Nachteil gereicht und wenn die Gründe fortgefallen sind, die ihn veranlaßt hatten, sein Versprechen zu geben. Wären alle Menschen gut, dann wäre diese Regel schlecht; da sie aber schlecht sind und ihr Wort dir gegenüber nicht halten würden, brauchst auch du dein Wort ihnen gegenüber nicht zu halten. Auch hat es noch nie einem Fürsten an rechtmäßigen Gründen gefehlt, um seinen Wortbruch zu verschleiern. ${ }^{98}$

Dementsprechend distanziert steht Machiavelli möglichen Bündnissen mit anderen Staaten gegenüber, die er nur dann zulassen will, wenn sie nicht der Machtposition des eigenen Staates schaden könnten; eine Haltung, welche ja durchaus auch in der zeitgenössischen Politik breite Anwendung fand, bedenkt man etwa die zahlreichen Bündniswechsel und Rechtsbrüche der Herrschaftszeit etwa Kaiser Maximilians und der Italienischen Kriege:

Hier ist zu bemerken, daß ein Fürst darauf achten muß, sich nie mit einem Mächtigeren zu verbünden, um andere anzugreifen, außer wenn ihn die Notwendigkeit dazu zwingt, wie oben gesagt wurde; siegt ihr nämlich, so wirst du sein Gefangener; Fürsten aber müssen, soweit sie können, vermeiden, in Abhängigkeit von anderen zu geraten. ${ }^{99}$

Dies gilt natürlich auch für Hilfstruppen, deren langfristigen Nachteile Machiavelli so hoch einschätzt, daß er sich ganz gegen das Prinzip von Bundeskontingenten ausspricht, sollten eigene Alternativen bestehen:

Solche Truppen können für ihren Eigentümer gut und nützlich sein, aber dem, der sie ruft, bringen sie fast immer Schaden; unterliegen sie nämlich, so bist du verloren, siegen sie hingegen, so bist du ihr Gefangener. ${ }^{100}$

Aus dem vorher Gesagten folgt mit zwingender Notwendigkeit, daß auch der Herrscher selbst die Skrupellosigkeit und den Willen zum unbedingten Machterhalt zu seiner obersten Tugend machen soll. Daher muß er nicht nur bedenken, daß viele moralische Tugenden sich in seiner gegenwärtigen Situation nachteilig auswirken können:

${ }_{98}$ Machiavelli, Principe 18: Non può, pertanto, uno signore prudente, né debbe, osservare la fede, quando tale osservanzia li torni contro e che sono spente le cagioni che la feciono promettere. E se gli uomini fussino tutti buoni, questo precetto non sarebbe buono; ma perché sono tristi, e non la osserverebbono a te, tu etiam non l'hai ad osservare a loro. Né mai a uno principe mancorono cagioni legittime di colorire la inosservanzia.

Dies schließt keineswegs eine systematische Parteinahme in Kriegsfällen zwischen zwei anderen Staaten aus: Machiavelli, Principe 21: E ancora stimato uno principe, quando egli è vero amico e vero inimico; cioè quando, sanza alcuno respetto, si scuopre in favore di alcuno contro ad un altro. Il quale partito fia sempre piú utile che stare neutrale; perché se dua potenti tuoi vicini vengono alle mani, o sono di qualità che, vincendo uno di quelli, tu abbi a temere del vincitore, o no. (Auch wird ein Fürst geachtet, wenn er ein wahrer Freund und ein wahrer Feind ist, das heißt, wenn er ohne irgendeinen Vorbehalt sich für den einen und gegen den anderen erklärt. Diese Entscheidung ist stets nützlicher, als neutral zu bleiben; wenn es nämlich zwischen zwei dir benachbarten Mächten zum Krieg kommt, dann stehen die Dinge so, daß du entweder, sobald einer von ihnen siegt, den Sieger zu fürchten hast oder nicht.)

${ }_{99}$ Machiavelli, Principe 21: E qui è da notare che uno principe debbe avvertire di non fare mai compagnia con uno piú potente di sé, per offendere altri, se non quando la necessità lo stringe, come di sopra si dice; perché, vincendo, rimani suo prigione: e li principi debbano fuggire, quanto possono, lo stare a discrezione di altri.

${ }^{100}$ Machiavelli, Principe 13: Queste arme possono essere utile e buone per loro medesime, ma sono, per chi le chiama, quasi sempre dannose; perché, perdendo, rimani disfatto: vincendom resti loro prigione. 
Auch darf es ihn dann nicht kümmern, in den Ruf solcher Laster zu geraten, ohne die er schwerlich seine Herrschaft bewahren könnte; wenn man nämlich alles genau betrachtet, wird man finden, daß manche Eigenschaft, die den Anschein der Tugend hat, bei ihrer Verwirklichung seinen Untergang herbeiführt, und daß manch andere, die den Anschein des Lasters hat, ihm bei ihrer Verwirklichung zu Sicherheit und Wohlbefinden verhilft. ${ }^{101}$

Er muß gleichzeitig auch bereit sein, nicht nur diese Tugenden zurückzustellen, ${ }^{102}$ sondern auch bewußt moralisch unakzeptable Handlungen begehen, um seine Macht zu erhalten; ein deutliches Indiz nicht nur für den unbedingten Pragmatismus Machiavellis, sondern auch dafür, wie wenig Überlegungen zum Seelenheil des Fürsten und zur göttlichen Fügung, welche der herkömmlichen Überzeugung nach über kurz oder lang doch immer moralisch gute Handlungen belohne, von Machiavelli noch in Betracht gezogen werden, während sie doch zumindest in den vorangehenden (und auch vielen der folgenden) Fürstenspiegeln ganz in den Mittelpunkt der Argumentation gestellt werden:

Für einen Fürsten ist es also nicht erforderlich, alle obengenannten guten Eigenschaften zu besitzen, wohl aber den Anschein zu erwecken, sie zu besitzen. Ich wage gar zu behaupten, daß sie schädlich sind, wenn man sie besitzt und ihnen stets treu bleibt; daß sie aber nützlich sind, wenn man sie nur $\mathrm{zu}$ besitzen scheint; so mußt du milde, treu, menschlich, aufrichtig sowie fromm scheinen und es auch sein; aber du mußt geistig darauf vorbereitet sein, dies alles, sobald man es nicht mehr sein darf, in sein Gegenteil verkehren zu können. ${ }^{103}$

\section{Schluß}

Wollen wir nach dieser notgedrungen sehr kurzen Übersicht über den geschichtlichen Kontext der mit den Namen Sūn Ž̌, Kauṭilya, Aineias Taktikos und Machiavelli verbundenen Werke sowie ihrer jeweiligen Aussagen über zwischenstaatliche Beziehungen und das damit verbundene Herrscherbild einen Vergleich wagen, so lassen sich folgende Überlegungen aufstellen. Allen vier Schriften ist gemein, daß sie nicht als moralische, rechtliche oder religiöse Idealschriften gedacht sind, sondern sich als reine „Fachliteratur“ verstehen, deren einziger oder doch wesentlicher Zweck die Optimierung eines bestimmten Verfahrens ist, losgelöst vom übergeordneten „Sinn“ dieses Verfahrens. Alle bislang gültigen Grundwerte der jeweiligen Gesellschaft werden in das dementsprechende Kalkül nur in Bezug auf ihren Nützlichkeitsfaktor einbezogen, also als Mittel zum Zweck oder als Hindernis zu seiner Verwirklichung; als Wert an sich aber haben sie keinerlei Einfluß mehr auf die innere Gestaltung des jeweiligen politischen oder militärischen Bereichs.

101 Machiavelli, Principe 15: Et etiam non si curi di incorrere nella infamia di quelli vizii sanza quali e'possa difficilmente salvare lo stato; perché, se si considerrà bene tutto, si troverrà qualche cosa che parrà virtú, e, seguendola, sarebbe la ruina sua; e qualcuna altra che parrà vizio, e, seguendola, ne riesce la securtà e il bene essere suo.

102 Zum Tugendbegriff Machiavellis vgl. Mansfield 1996; Mittermaier 2005.

103 Machiavelli, Principe 18: A uno principe, adunque, non è necessario avere in fatto tutte le soprascritte qualitá ma è bene necessario parere di averle. Anzi ardirò di dire questo, che, avendole e osservandole sempre, sono dannose; e parendo di averle, sono utili; come parere pietoso, fedele, umano, intero, religioso, ed essere; ma stare in modo edificato con l'animo, che, bisognando non essere, tu possa e sappi mutare el contrario. 
Das sich hieraus ergebende, teils skrupellose, teils desillusionierte, teils zynische Menschenbild schlägt sich nicht nur im hemmungslosen Einsatz selbst brutalster Machtmittel nieder, wenn die Behauptung der Herrschaft oder des Sieges dies notwendig macht, es führt auch zu einem Klima nahezu paranoiden Mißtrauens, das sich in fast endlosen Überlegungen zu Spionen, Verschwörungen, Attentaten und Umsturzversuchen aller Art niederschlägt. Dieses Mißtrauen anderen Menschen gegenüber muß langfristig auch Auswirkungen auf die Sichtweise der Beziehungen zwischen den Staaten haben: Freundschaft wie Feindschaft werden nunmehr allein auf Grundlage strategischer und machtpolitischer Konstellationen gehandhabt; Bündnisse im Schnitt eher als gefährlich denn als vorteilhaft betrachtet; Friedensverträge nur so lange als gültig gewertet, wie die ihnen zugrundeliegenden Bedingungen Gültigkeit haben, und sind bedenkenlos zu brechen, sobald dies einen Vorteil bietet. Es ist daher unausweichlich, daß das Herrscherbild, das sich aus dieser Konstellation ergibt, ebenfalls über gängigen moralischen Regeln zu stehen hat, die der Fürst oder Befehlshaber bestenfalls aus gesellschaftlicher Rücksicht zu befolgen hat, und auch dies nur so lange, wie seine Macht nicht bedroht ist: Für ein heroisches Scheitern durch Festhalten an inneren Werten oder gar für eine Hoffnung auf die weltimmanente Belohnung sittlicher Güte haben die Fachschriftsteller Sūn Ž̃, Kauțilya, Aineias Taktikos und Machiavelli im Gegensatz zu ihren Vorgängern keinerlei Verständnis mehr.

Nun könnte man diese hier sehr rasch skizzierten Gemeinsamkeiten ${ }^{104}$ im Ansatz (die natürlich die unausweichlichen Verschiedenheiten im Detail nicht verdecken sollen) leicht als nichtssagende oberflächliche Übereinstimmungen, ja vielleicht sogar als anthropologische Allgemeinheiten abtun, indem man entweder die vier verglichenen Schriften gegen andere, scheinbar gleichgelagerte Texte anderer Epochen ausspielt, um ihre Allgemeingültigkeit zu zeigen, oder die hier geäußerten oder doch implizierten Aussagen unter dem Blickwinkel als bloße Banalitäten betrachtet, Politik sei schon immer zumindest für die Herrschenden nichts anderes gewesen als ein Mittel, ihr jeweiliges Eigeninteresse in der einen oder anderen Weise mehr oder weniger skrupellos zu wahren und zu mehren. Nun sei durchaus nicht ausgeschlossen, daß sich auch zu anderen Zeiten und in anderen Hochkulturen analoge Positionen zu denen finden, die hier paradigmatisch für China, Indien, das antike Griechenland und das abendländische Italien behandelt wurden, gleichzeitig aber doch der starken Vermutung Ausdruck verliehen, daß jene weiteren denkbaren Texte sich wohl ebenfalls wieder einer ganz analogen historischen Konstellation wie die hier dargestellten Positionen verdanken und demgemäß den hier versuchsweise eröffneten Horizont möglicherweise zu erweitern, nicht aber fundamental umzustürzen geeignet sind. ${ }^{105}$

${ }^{104}$ Andere Gesichtspunkte würden es erlauben, die hier besprochenen Parallelen noch erheblich zu erweitern. Pars pro toto sei hier auf die ziemlich stereotype Rolle der Frauen hingewiesen: Bei Aineias $(40,4-5)$ dienen sie höchstens dazu, in männlicher Verkleidung den Gegner im Unklaren über die tatsächliche Truppenstärke zu lassen; von Sūn Ž̃ ist überliefert, er habe seine Fähigkeiten dadurch bewiesen, daß er „selbst Frauen“ militärisch vorbildlich gedrillt habe, und bei Kautilya sind Frauen ganz auf die Rolle von Spioninnen und Hetären reduziert, nicht unähnlich den Anspielungen bei Machiavelli.

${ }^{105}$ Der interessierte Leser sei hier auf meine diesbezüglichen Überlegungen in Engels 2018a und 2018b verwiesen. 
Was nun die angebliche anthropologische Permanenz skrupelloser Machtpolitik über Zeit und Raum hinaus betrifft, so sagt eine solche Vermutung in durchaus bedenklicher Weise nicht nur mehr über die eigene Lebenswelt und persönliche Einstellung dessen aus, der sie vertritt, als über die Realität der Vergangenheit, sie scheint auch vom faktischen Ansatz her fragwürdig zu sein. Es ist nämlich ein weiteres Ergebnis dieser kurzen Untersuchung, daß die offensichtlichen Analogien der vorgestellten Texte sich auch einer analogen historischen Situation verdanken und somit auf eine übergeordnete morphologische Dynamik verweisen. Freilich sei nicht bestritten, daß Skrupellosigkeit, Zynismus und Willen zur Macht seit jeher (und leider wohl auch auf immerdar) das Denken und Handeln einiger, manchmal sogar vieler Machtträger bestimmen und wohl auch hier und da im Laufe der Zeiten immer wieder schriftlich fixiert und systematisiert wurden. Die Allgegenwart eines Motivs bedeutet nun aber gerade nicht, daß der entsprechende Gedanke auch ,typisch“ für den Geist und die überwiegende Realität einer jeden Zeit ist, sondern nur, daß er manchmal verstärkt, manchmal aber auch nur sehr schwach faßbar ist, wie übrigens schon Machiavelli erkannt und am Beispiel der Klagen über die ,guten alten Zeiten“ exemplarisch formuliert hat:

Alle Menschen loben die Vergangenheit und beklagen die Gegenwart, und das oft ohne Grund. Sie sind solchermaßen von allem, was einst existierte, eingenommen, daß sie nicht nur jene Zeit loben, die sie doch nur durch die Darstellungen kennen, welche die Historiker hinterlassen haben, sondern, wenn sie alt sind, hört man sie oft damit angeben, was sie alles in ihrer Jugend gesehen haben. Ihre Meinung ist meistens fehlerhaft. [...] Aber es stimmt nicht, daß sie immer falsch ist. Tatsächlich nämlich ist es notwendig, daß unsere Urteile manchmal mit der Wahrheit übereinstimmen, im Einklang mit jener dauerhaften Bewegung aller menschlichen Dinge, welche ohne Rast entweder niedergehen oder aufsteigen. So sieht man zum Beispiel eine Stadt oder einen Staat eine Verfassung aus den Händen eines geschickten Gesetzgebers empfangen, dessen Talent sie für einige Zeit Fortschritte hin zur Vollkommenheit machen läßt. Jeder, der dann in diesem Staat lebt, aber eher die Vergangenheit als die Gegenwart lobt, irrt sich daher notwendigerweise; und der Grund seines Irrtums liegt in den oben besprochenen Argumenten. Aber wenn er in dieser Republik oder diesem Staat zu einer Zeit des Niedergangs lebt, dann irrt er nicht mehr [mit seinem Urteil]. ${ }^{106}$

Betrachtet man nun die offensichtliche Tatsache, daß die hier behandelten vier Schriften mit ihrer parallelen staatstheoretischen Ausrichtung nicht nur zu den jeweils ersten Texten in ihrem jeweiligen Kulturkreis zählen, die sich explizit mit den behandelten Fragen auseinandersetzen, sondern auch, daß sie dies in reichlich analoger Weise tun, und zudem, daß die historischen Rahmenbedingungen strukturell durchaus vergleichbar waren, drängt sich der Gedanke auf, daß wir es hier mit mehr als nur der rein akzidentellen Überlieferung von Texten zu tun haben, die lediglich eine omnipräsente und stets

106 Machiavelli, Discorsi II, praef.: Laudano sempre gli uomini, ma non sempre ragionevolmente, gli antichi tempi, e gli presenti accusano: ed in modo sono delle cose passate partigiani, che non solamente celebrano quelle etadi che da loro sono state, per la memoria che ne hanno lasciata gli scrittori, conosciute; ma quelle ancora che, sendo già vecchi, si ricordano nella loro giovanezza avere vedute. E quando questa loro opinione sia falsa [...]. Ma non essere gì̀ sempre vero che si erri nel farlo. Perché qualche volta è necessario che giudichino la verità; perché, essendo le cose umane sempre in moto, o le salgano, o le scendano. E vedesi una città o una provincia essere ordinata al vivere politico da qualche uomo eccellente, ed, un tempo, per la virtù di quello ordinatore, andare sempre in augumento verso il meglio. Chi nasce allora in tale stato, ed ei laudi più gli antichi tempi che i moderni, s'inganna; ed è causato il suo inganno da quelle cose che di sopra si sono dette. Ma coloro che nascano dipoi, in quella città o provincia, che gli è venuto il tempo che la scende verso la parte più ria, allora non s'ingannano. 
gleichstarke Tendenz in Worte fassen würden. Es ist vielmehr zu vermuten, daß gerade dieser dreifache „Zufall“ Indiz dafür ist, daß die hier formulierten Gedanken gleichzeitig so neuartig und auch typisch für die jeweilige Zeit waren, daß gerade jene Texte, die sie zum ersten Mal in dieser Vollständigkeit und Klarheit festhielten, sich über die Jahrhunderte hinweg als „klassischer“ Ausdruck reiner Machtpolitik erhalten haben und dementsprechend auch von uns als typisch, nicht akzidentell für den jeweiligen Zeitgeist zu behandeln sind.

Und tatsächlich: Entziehen wir die vier Schriften einer rein statischen Verortung und setzen sie in ihren breiteren, dynamischen Kontext, stellen wir fest, daß Sūn Zǐ, Kauṭilya, Aineias Taktikos und Machiavelli allesamt an einer bedeutsamen Schwelle in der Entwicklung ihrer jeweiligen Hochkultur standen, die geradezu einem dialektischen Umschlag gleichkam. Auf der einen Seite befinden sie sich, mal näher, mal ferner, geistig knapp jenseits einer Epoche, welche noch wesentlich von den letzten Ausläufern des Gedankens der Einheit von Glauben, Macht und Weltbild geprägt war, sei es die späte, unter Xuan und You noch einmal kurzfristig restaurierte Zhou-Monarchie, sei es die kurzlebige Maurya-Herrschaft in Indien, sei es die ältere Tyrannis in Griechenland mit ihren großartigen, zumindest ideologisch ganz Griechenland umspannenden Herrschern wie Peisistratos und dem darauffolgenden antipersischen Kampfbund, oder sei es die habsburgische Weltmonarchie Karls V.

Das Zerbrechen des Einheitsgedankens führt dabei nicht nur zum Entstehen einer kaum noch von übergeordneten Institutionen oder Überzeugungen geordneten Fülle neuer, sich beständig in wechselseitigen Bündnissen bekriegender Staatengebilde, sondern auch eines neuen Menschenbilds, das durch Rationalität, Individualismus, Wissenschaftlichkeit und Befreiung von äußeren Kräften geprägt ist; sei es in China das „Frühlings- und Herbst“-Zeitalter, in Indien die Śunga-Periode, in Griechenland die Zeit der Klassik und Spätklassik oder in Europa das Zeitalter der Frühen Neuzeit, also der Renaissance, des Barocks und der Aufklärung (vier Zeitalter, die übrigens nicht zufälligerweise in jeder Beziehung auch kunst- und geistesgeschichtlich als die „klassischen“ Zeitalter der jeweiligen Hochkultur gelten). Kein Wunder, daß in diesem Zeitalter der inneren Freiheit, welche nach außen hin aber noch ganz oder doch größtenteils durch die Form autoritär-absolutistischer Staatsgebilde ${ }^{107}$ geprägt war, sich eine pragmatische politisch-militärwissenschaftliche Fachliteratur herausbilden konnte, welche dem neuen Zeitgeist Ausdruck verlieh und ihn gleichzeitig auch seinerseits aktiv mitprägte - zumindest eine Zeitlang.

Denn nach etwa zwei bis drei Jahrhunderten - und dies ist dann der jenseitige Horizont, der von einigen der behandelten Schriftstellern zwar bereits erahnt, aber noch nicht erreicht wurde - sollte sich aus der beschriebenen Gemengelage ein neues staatliches Paradigma herausbilden, welches die bisherigen, kleinteiligen und im stetigen Wandel befindlichen Strukturen in das Gleichgewicht, größerer, schwerfälligerer Machtgebilde

${ }^{107}$ Dies gilt explizit auch für die griechische Staatenwelt, die in jener Zeit zwar auf den ersten Blick von einer Fülle verschiedenster Verfassungsformen geprägt war, diese alle aber (im Gegensatz zur früheren und späteren Zeit) so absolut setzte, daß Spengler die klassische Demokratie nicht ganz zu Unrecht als „Absolutismus des Demos“ verstanden hat; eine Perspektive, die durch den Blick auf die frühneuzeitliche Situation ja nur bestätigt wird, wo die Republik der Vereinigten Niederlande in vielerlei Hinsicht in kaum weniger ,absolutistischer“ Form agierte als etwa Ludwig XIV. 
überführte, deren wechselseitigen Konflikte eine derartige Zerstörungskraft entfalteten, daß an das Fortsetzen der bisherigen Kabinetts- und Condottiere-Politik nicht mehr zu denken war; man erinnere sich hier nur an die schrecklichen Verwüstungen der Kriege zwischen den chinesischen „Kämpfenden Staaten“, den indischen Großreichen der ersten beiden nachchristlichen Jahrhunderte, der hellenistischen Mächte im griechischen Raum oder der europäischen Kolonialreiche des imperialistischen Zeitalters. Auch der Herrscher selber, zunächst in seinen Machtbefugnissen in jeder Hinsicht noch reichlich unbestimmt, sollte sich zunehmend zum Haupt einer komplexen Verwaltung entwickeln, in welcher Recht und Berechenbarkeit und nicht mehr fürstliche Willkür oberstes Effizienzprinzip sein mußten, so daß auch die Macht des Herrschers in feste Bahnen überführt wurde. Gleichzeitig bewirkte die immer größere Ausdehnung der jeweiligen Reiche in diesem Zeitalter, daß auch das alte Konzept der beliebigen Angliederung fremder Gebiete zunehmend der Stabilität ethnisch oder geographisch mehr oder weniger fest definierter Staaten wich (die sieben chinesischen Nationen, die indischen Großstaaten, die griechischen und italischen Bundes- und Territorialstaaten, die europäischen Nationalstaaten), was an die Stelle des skrupellosen „Jeder gegen jeden“ das nicht minder gefährliche, sicherlich aber berechenbarere Konzept der (freilich je nach Kulturkreis immer verschiedenartig verstandenen) „Nation“ setzte. Nunmehr waren es vielmehr Denker vom Format eines Sūn Bìn (孫臏) ${ }^{108}$ bzw. eines Mòzǐ (墨子, 479-392), ${ }^{109}$ eines Manu (dem angeblichen Verfasser des sicherlich eher kollektiv in den ersten nachchristlichen Jahrhunderten zusammengestellten Manusmrti), ${ }^{110}$ eines Asklepiodotos (1. Jh. v.Chr.) oder eines Clausewitz ${ }^{111}$ (1780-1831) bzw. eines de Jomini (1779-1869), welche die politisch-militärwissenschaftliche Fachliteratur beherrschten und zwar sicherlich einige der auch bei unseren Autoren faßbaren Züge übernahmen, sie aber doch in einen ganz anderen historischen und auch weltanschaulichen Kontext überführten: An die Stelle der landesfürstlichen Raubkriege traten der Gedanke des Verwaltungsstaats, des Wettrüstens, der erneuten gesellschaftlichen Stratifizierung und des Machtgleichgewichts als ultimativen (und paradoxen) Wahrers von Frieden und Wohlstand - eine Hoffnung, die in jeder einzelnen dieser Kulturen durch eine Reihe schrecklicher Weltkriege zunichte gemacht werden sollte, aus denen dann schließlich ein neues, definitives Universalreich hervorging. ${ }^{112}$

${ }^{108}$ Zur größeren moralistischen Tendenz des Sūn Bìn vgl. Lau - Ames 2003, 29-30.

109 S. einführend zum moralisierenden Militärdenken der Mohisten: Yates 1979.

${ }_{110}$ Zur Wechselbeziehung zwischen Arthaśästra und Manusmrti vgl. Nagarajan 1992; Olivelle 2004.

$111 \mathrm{Zu}$ dieser Kontinuität s. etwa Heuser 2014.

$112 \mathrm{Zu}$ den diesbezüglichen Parallelen zwischen China, Indien, Iran und der Antike vgl. Engels 2017a und Engels 2018c. Zu den Aussichten auf die sich zwar schon deutlich abzeichnende, aber faktisch noch nicht erfüllte europäische Zukunft vgl. Engels 2014. 


\section{LITERATURVERZEICHNIS}

Agrawal, A. (1989), Rise and Fall of the Imperial Guptas, Delhi.

Albertini, R. von (1955), Das florentinische Staatsbewußtsein im Übergang von der Republik zum Prinzipat, Bern.

Ames, R.T. (1993), Sun-tzu: The Art of Warfare: A New Translation Incorporating the Recently Discovered Yin-ch'üeh-shan Texts, New York.

Ames, R.T. (1994), The Art of Rulership. A Study in Ancient Chinese Political Thought, Albany.

Anderson, J. (1970), Greek Military Theory and Practice in the Age of Xenophon, Berkeley.

Baron, H. (1955), The Crisis of the Early Italian Renaissance: Civic Humanism and Republican Liberty in an Age of Classicism and Tyranny, 2 Bde., Princeton, NY.

Bauer, W. (2001), Geschichte der chinesischen Philosophie. Konfuzianismus, Daoismus, Buddhismus, München.

Bengtson, H. (1962), Die griechische Polis bei Aeneas Tacticus, Historia 11: 458-468.

Berridge, G.R., Keens-Soper, M., Otte, T.G. (Hgg.) (2001), Diplomatic Theory from Machiavelli to Kissinger, Basingstoke-New York.

Béteille, A. (1996), Varna and Jati, Sociological Bulletin 45.1: 15-27.

Bhargava, P.L. (1996), Chandragupta Maurya: A Gem of Indian History, New Delhi.

Bock, G., Skinner, Q., Viroli, M. (Hgg.) (1990), Machiavelli and Republicanism, Cambridge.

Boesche, R. (2002a), The First Great Political Realist: Kautilya and his Arthashastra, Lanham.

Boesche, R. (2002b), Moderate Machiavelli? Contrasting "The Prince" with Arthshastra of Kautilya, Critical Horizons 3.2: 253-276.

Brodersen, K. (2017), Aineias / Aeneas Tacticus. Stadtverteidigung, Berlin-Boston.

Brown, T. (1981), Aeneas Tacticus, Herodotus and the Ionian Revolt, Historia 30: 385-393.

Buck, A. (1985), Machiavelli, Darmstadt.

Burckhardt, J. (1860/1976), Die Kultur der Renaissance in Italien (1860), Stuttgart.

Capponi, N. (2010), An Unlikely Prince: The Life and Times of Machiavell, Cambridge.

Celato, S. (1968a), Enea Tattico. Il problema dell'autore e il valore dell'opera dal punto di vista militare, Atti e Memorie dell'Accademia Patavina 80: 53-67.

Celato, S. (1968b), La Grecia del IV secolo a. C. nell'opera di Enea Tattico, Atti e Memorie dell'Accademia Patavina 80: 215-244.

Celenza, Chr.S. (2015), Machiavelli: A Portrait, Cambridge, Mass.

Chabod, F. (1965), Scritti su Machiavelli, Torino.

Cheng, A. (2002), Histoire de la pensée chinoise, Paris.

Ching, J. (1993), Chinese Religions, London.

Choudhary, R.K. (1971), Kautilya's Political Ideas and Institutions, Varanasi.

Connell, W.J. (2015), Machiavelli nel Rinascimento italiano, Milano.

Coomaraswamy, A.K. (1942), Spiritual Authority and Temporal Power in the Indian Theory of Government, New Haven.

Das, S. (1994), Social Life in Ancient India: 800 BC-183 BC, Delhi.

David, E. (1986), Aeneas Tacticus, 11.7-10 and the Argive Revolution of 370 B.C., AJPh 107: 343-349.

Denecke, W. (2011), The Dynamics of Masters Literature: Early Chinese Thought from Confucius to Han Feizi, Cambridge.

Dikshitar, V.R.R. (1927), Kautalya and Machiavelli, Indian Historical Quarterly 3: 176-180.

Drekmeier, Ch. (1962), Kingship and Community in Early India, Stanford, CA.

Dumont, L. (1966), Le système des castes et ses implications, Paris.

Eisenhofer, H. (Übers.) (2014), Sunzi. Die Kunst des Krieges, Hamburg.

Engels, D. (2014), Auf dem Weg ins Imperium, Berlin-München. 
Engels, D. (2017a), Construction de normes et morphologie culturelle. Empire romain, chinois, sasanide et fatimide - une comparaison historique, in: T. Itgenshorst, Ph. LeDoze (Hgg.), La norme sous la République romaine et le Haut Empire. Élaboration, diffusion et contournements, Bordeaux: 53-73.

Engels, D. (2017b), 89. L'Ab Urbe condita, laboratoire de la pensée politique de Machiavel, in: P. Assenmaker (Hg.), Tite-Live. Une histoire de livres. 2000 ans après la mort du Prince des historiens latins, Namur: 127-133.

Engels, D. (2018a), Kulturmorphologie und Willensfreiheit. Überlegungen zu einer neuen komparatistischen Geschichtsphilosophie, in: D. Engels, M. Otte, M. Thöndl (Hgg.), 100 Jahre „,Der Untergang des Abendlandes “, Waltrop: 79-101.

Engels, D. (2018b), Spengler im 21. Jahrhundert. Überlegungen und Perspektiven zu einer Überarbeit der Spengler'schen Kulturmorphologie, in: S. Fink, R. Rollinger (Hgg.), Oswald Spenglers Kulturmorphologie - eine multiperspektivische Annäherung, Berlin: 451-486.

Engels, D. (2018c), ,Reinheit‘ als Herrscherqualität im ideologischen Narrativ der großen Universalhistoriker der alten Welt, in: L. Clemens, B. Eckhardt, K. Zimmermann (Hgg.), Purity and Authority, Leiden.

Engels, D., Geis, L., Kleu, M. (Hgg.) (2010), Zwischen Ideal und Wirklichkeit: Herrschaftsausübung auf Sizilien von der Antike bis zum Spätmittelalter, Stuttgart.

Falkenhausen, L. von (2006), Chinese Society in the Age of Confucius, Los Angeles.

Frolov, E. (1974), Das Problem der Monarchie und der Tyrannis in der politischen Publizistik des 4. Jahrhunderts v.u.Z., in: E.C. Welskopf (Hg.), Hellenische Poleis. Krise - Wandlung - Wirkung, Darmstadt: 401-434.

Garin, E. (1993), Machiavelli fra politica e storia, Torino.

Gautam, P.K. (2013), One Hundred Years of Kautilya's Arthashastra, Delhi.

Gehrke, H.-J. (1985), Untersuchungen zu den inneren Kriegen in den griechischen Staaten des 5. und 4. Jahrhunderts v. Chr., München.

Gehrke, H.-J. (1986), Jenseits von Athen und Sparta. Das dritte Griechenland und seine Staatenwelt, München.

Gennaro S. (1965), Niccolò Machiavelli. Geschichte seines politischen Denkens, Stuttgart u.a.

Gilbert, A. (1938), Machiavelli's Prince and Its Forerunners, Durham.

Godman, P. (1998), From Poliziano to Machiavelli: Florentine Humanism in the High Renaissance, Princeton.

Goez, W. (1984), Grundzüge der Geschichte Italiens in Mittelalter und Renaissance, Darmstadt.

Graham, A.C. (1993), Disputers of the Tao: Philosophical Argument in Ancient China, La Salle.

Günther, Sv. (2014), Framing the Financial Thoughts of Aeneas Tacticus: New Approaches of Theory to Economic Discourses in Antiquity, JAC 29: 77-86.

Gupta, D. (1980), From Varna to Jati: The Indian Caste System, from the Asiatic to the Feudal Mode of Production, Journal of Contemporary Asia 10.3: 249-271

Hale, J.R. (1961), Machiavelli and Renaissance Italy, New York.

Hanson, V.D. (1989), The Western Way of War: Infantry Battle in Classical Greece, New York.

Hanson, V.D. (Hg.) (1991), Hoplites: The Classical Greek Battle Experience, London-New York.

Hay, D. (1977), The Italian Renaissance in Its Historical Background, Cambridge.

Heuser, B. (2014), The Strategy Makers: Thoughts on War and Society from Machiavelli to Clausewitz, Santa Monica, CA.

Hoeges, D. (2000), Niccolò Machiavelli. Die Macht und der Schein, München.

Horkheimer, M. (1971), Anfänge der bürgerlichen Geschichtsphilosophie, Frankfurt a.M.

Hörnqvist, M. (2004), Machiavelli and Empire, Cambridge.

Hsu, Ch. (1965), Ancient China in Transition: An Analysis of Social Mobility, 722-222 BC, Stanford.

Hudson-Williams, T. (1904), The Authorship of the Greek Military Manual Attributed to Aeneas Tacticus, AJPh 25: 390-405.

Hug, A. (1877), Aineias von Stymphalos, ein arkadischer Schriftsteller aus classischer Zeit, Zürich.

Hunter, L.W., Handford, S.A. (1927), Aineiou Poliorketika, Oxford. 
Indra (1957), Ideologies of War and Peace in Ancient India, Hoshiarpur.

Jurdjevic, M. (2014), A Great and Wretched City: Promise and Failure in Machiavelli's Florentine Political Thought, Cambridge, MA.

Kangle, R.P. (1969), Kautilya Arthashastra, 3 Bde., Delhi.

Konow, S. (1945), Kautalya Studies, Delhi.

Kühnhardt, L. (1988), Staatsordnung und Macht in indischer Perspektive - Kautilya Chanakya als Klassiker der politischen Ideengeschichte, HZ 247: 333-355.

Lagerwey, J. (Hg.) (2004), Religion and Chinese Society, 2 Bde., Paris.

Lagerwey, J., Kalinowski, M. (Hgg.) (2008), Early Chinese Religion: Part One: Shang through Han (1250 BC-220 AD), 2 Bde., Leiden.

Landon, W.J. (2005), Politics, Patriotism and Language: Niccolò Machiavelli's "Secular Patria" and the Creation of an Italian National Identity, New York.

Lange, A.C. (1879), De Aeneae commentario poliorcetico, Berlin.

Lau, D.C. (1965), Some Notes on the Sun Tzu, Bulletin of the School of Oriental and African Studies 18: 318-335.

Lau, D.C., Ames, R.T. (2003), Sun Bin. The Art of Warfare: A Translation of the Classic Chinese Work of Philosophy and Strategy, New York.

Lau, D.C., Ching, Ch.F. (Hgg.) (1992), A Concordance to the Militarists (Sunzi, Yuliaozi, Wuzi, Simafa), Hong Kong.

Lehmann, G.A. (1980), Krise und innere Bedrohung der hellenischen Polis bei Aeneas Tacticus, in: Studien zur antiken Sozialgeschichte. FS Friedrich Vittinghoff, Köln: 71-86.

Lehmann, G.A. (1989), Aeneas Tacticus und die politisch-soziale Krise der zeitgenössischen PolisWelt, Index 17: 105-115.

Li, X. (1985), Eastern Zhou and Qin Civilization, New Haven-London.

Li, Y. (Hg.) (1977), Shang Yang's Reforms and State Control in China, White Plains.

Lintott, A.W. (1982), Violence, Civil Strife and Revolution in the Classical City, 850-330 BC, LondonCanberra.

Loewe, M. (1977), Manuscripts Found Recently in China: A Preliminary Survey, TP 63.2: 99-136.

Loewe, M., Shaughnessy, E.L. (Hgg.) (1999), The Cambridge History of Ancient China: From the Origins of Civilization to 221 BC, Cambridge.

Mabbett, I. (2010), The Kautiliya Arthasastra and the Concept of Secularism, Journal of South Asian Studies 33: 13-32.

Mair, V.H. (2007), The Art of War: Sun Zi's Military Methods, New York.

Mansfield, H.C. (1996), Machiavelli's Virtue, Chicago, Il.

Marinovich, L.P. (1962), The Sociopolitical Struggle and the Use of Mercenaries in Fourth-Century BC Greece in the Treatise of Aeneas Tacticus (auf Russisch), VDI 81: 49-77.

McClish, M. (2009), Political Brahmanism and the State: A Compositional History of the Arthaśāstra, Austin.

McNeal, R. (2000), Acquiring People: Social Organization, Mobilization, and Discourse on the Civil and the Martial in Ancient China, Washington.

Meyer, J.J. (1926), Das altindische Buch vom Welt - und Staatsleben: Das Arthacastra des Kautilya, Leipzig.

Meyer, J.J. (1927), Über das Wesen der altindischen Rechtsschriften und ihr Verhältnis zu Kautilya, Leipzig.

Mittermaier, K. (2005), Machiavelli. Moral und Politik zu Beginn der Neuzeit, Gernsbach.

Mosley, D.J. (1973), Envoys and Diplomacy in Ancient Greece, Stuttgart.

Mukherjee, Bh. (1976), Kautilya's Concept of Diplomacy, Columbia.

Münkler, H. (1982), Machiavelli. Die Begründung des politischen Denkens der Neuzeit aus der Krise der Republik Florenz, Frankfurt a.M.

Nadeau, R.L. (Hg.) (2012), The Wiley-Blackwell Companion to Chinese Religions, Malden, MA.

Nag, K.D. (1923), Les théories diplomatiques de l'Inde ancienne et l'Arthaçâstra, Paris. 
Nagarajan, V. (1992), Evolution of the Social Polity of Ancient India from Manu to Kautilya, Nagpur. Najemy, J. (Hg.) (2010), The Cambridge Companion to Machiavelli, Cambridge.

Narain, V. (2005), Kauțilīya Arthaśāstra: Sanskrit, Transliteration, and English Translation with an Exhaustive Introduction (auf Basis der Übersetzung von R. Shamasastry), Delhi.

Nilakanta Sastri, K.A. (Hg.) (1967), Age of the Nandas and Mauryas, Delhi.

Olivelle, P. (2004), Manu and the Arthaśāstra, A Study in Sāstric Intertextuality, Journal of Indian Philosophy 32: 281-291.

Olivelle, P. (2006), Between the Empires: Society in India 300 BCE to 400 CE, Oxford.

Olivelle, P. (2013), King, Governance, and Law in Ancient India: Kautilya's Arthaśāstra, Oxford.

Oppenheimer, P. (2011), Machiavelli: A Life Beyond Ideology, London-New York.

Pankenier, D.W. (2013), Astrology and Cosmology in Early China, Cambridge.

Paret, P. (ed.) (1986), Makers of Modern Strategy from Machiavelli to the Nuclear Age, Princeton.

Parmar, A. (1987), Techniques of Statecraft: A Study of Kautilya's Arthashastra, Delhi.

Parsons, W.B. (2016), Machiavelli's Gospel: The Critique of Christianity in The Prince, Rochester.

Pines, Y. (2002), Foundations of Confucian Thought: Intellectual Life in the Chunquiu Period, Honolulu.

Pines, Y. (2009a), Imagining the Empire? Concepts of 'Primeval Unity' in Pre-Imperial Historiographic Tradition, in: F. Mutschler, A. Mittag (Hgg.), Conceiving the Empire: China and Rome Compared, Oxford: 67-90.

Pines, Y. (2009b), Envisioning Eternal Empire: Chinese Political Thought of the Warring States Era, Honolulu.

Pines, Y. (2010), Political Mythology and Dynastic Legitimacy in the Rong Cheng Shi Manuscript, Bulletin of SOAS 73.3: 503-529.

Pöhlmann, R. von (1925), Geschichte der sozialen Frage und des Sozialismus in der antiken Welt, München, 2 Bde. (3. Aufl.), München.

Pregadio, F. (Hg.) (2013), The Encyclopedia of Taoism, 2 Bde., London.

Pullan, Br. (1973), History of Early Renaissance Italy, London.

Rangarajan, L.N. (1992), Kautilya: The Arthashastra, London.

Raphals, L. (1992), Knowing Words: Wisdom and Cunning in the Classical Traditions of China and Greece, Ithaka, NY.

Raychaudhuri, H. (1927), Political History of Ancient India, New Delhi (2. Aufl.).

Ridolfi, R. (1972), Vita di Niccolo Machiavelli, Firenze (5. Aufl.).

Rippel, Ph. (ed. und übers.) (1986), Niccolò Machiavelli. Il Principe. Der Fürst. Italienisch/Deutsch, Stuttgart.

Ritschl, E., Schetlich, M. (1973), Studien zum Kautilya Arthasastra, Berlin.

Rossi Leidi, Th. (2015), L'histoire cyclique. Machiavel et l'aristotélisme de la Renaissance, in: D. Engels (Hg.), Von Platon bis Fukuyama, Bruxelles: 160-180.

Roy, G.J. (1981), Diplomacy in Ancient India, Patna.

Ruggiero, G. (2015), The Renaissance in Italy: A Social and Cultural History of the Rinascimento, Cambridge.

Sawyer, R.D. (2007), The Seven Military Classics of Ancient China, Beijing.

Scharfe, H. (1968), Untersuchungen zur Staatsrechtslehre des Kautalya, Wiesbaden.

Schlingloff, D. (1967), Arthashastra-Studien, Wiener Zeitschrift für die Kunde Süd- und Ostasiens 11: 44-80.

Schrenck-Notzing, C. von (Hg.) (1978), Konservative Köpfe von Machiavelli bis Solschenizyn, München.

Schulz, R. (1999), Militärische Revolution und politischer Wandel. Das Schicksal Griechenlands im 4. Jh. v.Chr., HZ 268: 281-310.

Schwartz, E. (1894), s.v. Aineias (3), RE 1,1: 1019-1021.

Sharma, R.Sh. (1954), Superstition and Politics in the Arthashastra of Kautilya, Journal of the Bihar Research Society 40.3: 223-231. 
Sharma, R.Sh. (1959), Aspects of Political Ideas and Institutions in Ancient India, Delhi.

Sil, N.P. (1983), Kautilya's Arthasastra and Machiavellism: A Reevaluation, Quarterly Review of Historical Studies 23.2

Sil, N.P. (1985a), Kauțilya's Arthaśāstra: A Comparative Study, New York.

Sil, N.P. (1985b), Political Morality and Political Necessity: Kauṭilya and Machiavelli Revisited, Journal of Asian History 19: 101-142.

Sil, N.P. (2017), The Analect and the Arthaśāstrsa: Kongzi of Zhou China and Kautilya of Maurya India Compared (http://journals.sagepub.com/doi/abs/10.1177/2158244017747324).

Singh, G.P. (2003), Republics, Kingdoms, Towns and Cities in Ancient India, New Delhi.

Singh, U. (2015), A History of Ancient and Early Medieval India, New Delhi.

Spengler, J.J. (1969), Kautilya, Plato and Lord Shang, Comparative Political Economy 113.6: 450-457.

Sternbach, L. (1973), Bibliography of Kautiliya Arthasastra, Hoshiarpur.

Strauss, L. (1958), Thoughts on Machiavelli, Chicago, Il.

Sullivan, V.B. (1996), Machiavelli's Three Romes: Religion, Human Liberty, and Politics Reformed, Chicago, Il.

Tejada, J.V. (2004), Warfare, History and Literature in the Archaic and Classical Periods, Historia 53: $129-146$.

Trautmann, Th.R. (1971), Kauțilya and the Arthaśāstra: A Statistical Investigation of the Authorship and Evolution of the Text, Leiden.

Trautmann, Th.R. (2012), Arthashastra: The Science of Wealth, New Delhi.

Tuplin, C.J. (1976), Aeneas Tacticus. Poliorketika, 18.8, LCM: 127-131.

Unger, M.J. (2011), Machiavelli: A Biography, New York.

Urban, R. (1986), Zur inneren und äußeren Gefährdung griechischer Städte bei Aeneas Tacticus, in: Studien zur alten Geschichte: Siegfried Lauffer zum 70. Geburtstag, Rom: 989-1002.

Vandermeersch, L. (1965), La Formation du légitisme, Paris.

Vela Tejada, J. (1991), Estudio sobre la lengua de la Poliorcética de Eneas el Táctico, Zaragoza.

Vela Tejada, J. (1992), Eneas el Táctico come testimonio histórico, in: Homenatge a Josep Alsina, Bd. I, Tarragona: 353-358.

Vela Tejada, V. (1993), Tradición y originalidad en la obre de Eneas el Táctico : la génesis de la historiografia militar, Minerva 7: 72-92.

Viroli, M. (1998), Machiavelli, Oxford.

Viroli, M. (1999), Repubblicanesimo, Roma-Bari.

Viroli, M. (2005), Il Dio di Machiavelli e il problema morale dell'Italia, Roma-Bari.

Vorländer, K. (1926), Von Machiavelli bis Lenin. Neuzeitliche Staats- und Gesellschaftstheorien, Leipzig.

Weibel, E. (2012), Machiavel. Biographie politique, Paris.

Whitehead, D. (1992), L'image de l'étranger dans la Poliorcétique d'Énée le Tacticien, in: R. Lonis (Hg.), L'Étranger dans le monde grec, Bd. II, Nancy: 315-331.

Whitehead, D. (2002), Aineias the Tactician: how to Survive under Siege. A Historical Commentary, Oxford (2., rev. Auflage).

Winterling, A. (1991), Polisbegriff und Stasistheorie des Aeneas Tacticus. Zur Frage der Grenzen der griechischen Polisgesellschaften im 4. Jahrhundert v.Chr., Historia 40: 193-229.

Wu, T. (2016), Public Festivals, Political Manipulations and Civil Strife. Aeneas Tacticus on Rituals and City Defenses, Marburger Beiträge 34: 41-52.

Yates, R.D.S. (1979), The Mohists on Warfare. Technology, Technique and Justification, Journal of the American Academy of Religion 47: 549-603.

Zaman, R.U. (2006), Kautilya: The Indian Strategic Thinker and Indian Strategic Culture, Comparative Strategy 25.3: 231-247. 